Draft version April 22, 2019

Preprint typeset using LATEX style AASTeX6 v. 1.0

\title{
GRAVITATIONAL-WAVE MERGING EVENTS FROM THE DYNAMICS OF STELLAR MASS BINARY BLACK HOLES AROUND THE MASSIVE BLACK HOLE IN A GALACTIC NUCLEUS
}

\author{
FUPENG ZHANG ${ }^{1,2}$, LiJing ShAO ${ }^{3}$, Weishan ZhU ${ }^{2}$ \\ ${ }^{1}$ School of Physics and Electronic Engineering, Guangzhou University, 510006 Guangzhou, China, zhangfupeng@gzhu.edu.cn \\ ${ }^{2}$ School of Physics and Astronomy, Sun Yat-Sen University, Guangzhou 510275, China \\ ${ }^{3}$ Kavli Institute for Astronomy and Astrophysics, Peking University, Beijing 100871, China
}

\begin{abstract}
We study the dynamical evolution of the stellar mass binary black holes (BBHs) in a galactic nucleus that contains a massive black hole $(\mathrm{MBH})$. For a comprehensive study of their merging events, we consider simultaneously the non-resonant and resonant relaxations of the BBHs, the binary-single encounters of the BBHs with the field stars, the Kozai-Lidov (KL) oscillation and the close encounters between the BBHs and the central $\mathrm{MBH}$, which usually lead to binaries' tidal disruptions. As the BBHs are usually heavier than the background stars, they sink to the center by mass segregation, making the KL oscillation an important effect in merging BBHs. The binary-single encounters can not only lead to softening and ionization of the BBHs, it can also make them hardening, that increases the merging rates significantly. The mergers of BBHs are mainly contributed by galaxies containing MBHs less massive than $10^{8} M_{\odot}$ and the total event rates are likely in orders of $1-10 \mathrm{Gpc}^{-3} \mathrm{yr}^{-1}$, depending on the detailed assumptions of the nucleus clusters. About $3-10 \%$ of these BBH mergers are with eccentricity $\geq 0.01$ when their gravitational wave oscillating frequencies enter the LIGO band $(10 \mathrm{~Hz})$. Our results show that merging the BBHs within galactic nuclei can be an important source of the merging events detected by the Advanced LIGO/Virgo detectors, and they can be distinguished from BBH mergers from the galactic fields and globular clusters when enough events are accumulated.

Keywords: Black-hole physics - gravitation - Galaxy: center - Galaxy: nucleus - relativistic processes - stars: kinematics and dynamics
\end{abstract}

\section{INTRODUCTION}

The first gravitational-wave (GW) event GW150914 has been discovered by the Advanced LIGO (Abbott et al. 2016a). It is caused by the merger of a stellar mass binary black hole (BBH) with component masses given by $\sim 36 M_{\odot}$ and $\sim 29 M_{\odot}$. Later on, a number of stellar-mass $\mathrm{BBH}$ mergers were reported (for the latest results see Abbott et al. 2018), e.g., GW151226 (Abbott et al. 2016c), GW170104 (Abbott et al. 2017a), GW170608 (Abbott et al. 2017b), and GW170814 (Abbott et al. 2017c). Currently, the locations of these merging events and corresponding mechanisms remain largely uncertain. The proposed explanations include (1) merging $\mathrm{BBHs}$ in the isolated environments (e.g., Dominik et al. 2015; Belczynski et al. 2016); (2) merging BBHs in the globular clusters (e.g., Morscher et al. 2015; Rodriguez et al. 2015, 2016a; Fragione \& Kocsis 2018); (3) merging BBHs in the galactic nuclei. For example, the GW capture of two encountering black holes (O'Leary et al. 2009), and the merging of BBHs due to the Kozai-Lidov (KL) effect (e.g., Antonini et al. 2010; Antonini \& Perets 2012; VanLandingham et al. 2016; Petrovich \& Antonini 2017). Such merging events can be possible without the presence of a central massive black hole (MBH) (Miller \& Lauburg 2009; Antonini \& Rasio 2016). So far, it is still challenging to distinguish these different mechanisms from the observations, as localizations are poor, and that electromagnetic counterparts for these events have not been found convincingly.

A galactic nucleus that harbors a $\mathrm{MBH}$ in the center is a very complex system. Both the inner (orbit of the $\mathrm{BBH}$ circling with each other) and outer (orbit of the $\mathrm{BBH}$ circling the central $\mathrm{MBH}$ ) orbits within such environments can be affected by a number of different dynamical effects: (1) The dynamical evolution of the outer orbit of the BBHs, due to the non-resonant (NR, or two-body relaxation) or resonant relaxation (RR) effects (Rauch \& Tremaine 1996); (2) The encounters between the BBHs and field stars (or any stellar remnants). Such processes can change the inner or- 
bit of the BBHs, and lead to softening or hardening of the binary, or even exchange of the binary components with the incoming objects (e.g., Heggie \& Hut 1993; Sigurdsson \& Phinney 1993; McMillan \& Hut 1996; Downing et al. 2010; Samsing et al. 2014); (3) A $\mathrm{MBH}$ and a $\mathrm{BBH}$ form a natural hierarchical triple system, where the KL effects will lead to the orbital oscillations (e.g., Kozai 1962; Naoz et al. 2013; Naoz 2016). In some cases the eccentricity of the BBH can be excited to an extremely high value such that the $\mathrm{BBH}$ will merge eventually due to the GW radiation (e.g. Antonini \& Perets 2012; Hoang et al. 2018); (4) The tidal disruption of the BBHs by the central MBH. The strong tidal force of the central $\mathrm{MBH}$ will likely disrupt a $\mathrm{BBH}$ that is very close to it. Along the path that leads to the merging of the BBHs, all of the above dynamical processes may affect the evolution of the inner and outer orbits. Thus, to obtain a realistic estimation of the merging rates, all of the above dynamical processes should be simultaneously considered. However, they have not been well combined in previous studies yet.

We notice that the BBHs in a galactic nucleus are most likely soft binaries (Hopman 2009) and to be merged within the galactic nucleus, different from those BBHs in globular clusters, which are mostly hard binaries and likely to be merged after they are ejected from the cluster (Rodriguez et al. 2016a). Some dynamical processes, e.g., KL effects, RR, tidal disruptions of $\mathrm{BBH}$ sy the central $\mathrm{MBH}$, are unique, requiring the existence of a $\mathrm{MBH}$, and they happen only in the galactic nuclei. Thus, to distinguish the mergers of stellar mass $\mathrm{BBHs}$ that take place in galactic nuclei and those resulting from globular clusters, it is important for us to trace all of the dynamical effects in a galactic nucleus mentioned above.

Here in this work, we aim to build a comprehensive framework to study the evolution of stellar mass BBHs in a galactic nucleus, for both the inner and the outer orbits, by using Monte-Carlo methods that include all of the above effects. Such a framework can provide a more detailed estimation of how the merging rate of BBHs is affected by individual, or combinations of these effects. To investigate the importance of the mergers of BBHs in galactic nuclei for the Advanced LIGO/Virgo detectors, we can compare our predicted merging signals and merging rates in the local universe with observations. Our investigation can not only improve the understandings of the channels of BBHs merging in a galactic nucleus, but also the dynamics of stars and binaries around the $\mathrm{MBH}$.

The paper is organized as follows. We describe the simulation details of various dynamical processes in Section 2. In Section 3 we perform a number of Monte-Carlo simulations to show the effects of various dynamical processes on the merging and evolution of the BBHs. In Section 4 we provide estimations of the merging rates of stellar mass BBHs in a galactic nucleus in the nearby universe. Section 3 concentrates mostly on the details of the BBH's dynamical evolution in a galactic nucleus, thus readers who are more interested in the rate and properties of the merging events of BBHs in the local universe, can skip Section 3 and turn to Section 4 directly. Discussion and conclusion are provided in Sections 5 and 6 , respectively. For clarity, some notations of variables that are frequently used in this paper are summarized in Table 1.

\section{THE METHOD}

We consider a nucleus cluster that consists of field stars with the same mass $m_{\star}$, and contains a MBH in the center with mass $M_{\bullet}$. Then the MBH dominates the dynamical evolutions of the cluster within the gravitational influence radius $r_{h}$, given by (Hopman \& Alexander 2006)

$$
r_{h}=\frac{G M_{\bullet}}{\sigma_{h}^{2}}=2.31 \mathrm{pc}\left(\frac{M_{\bullet}}{4 \times 10^{6} M_{\odot}}\right)^{1 / 2},
$$

where $\sigma_{h}$ is the velocity dispersion of the galaxy, and we have used the well-established $M_{\bullet}-\sigma$ relation.

The enclosed mass within $r_{h}$ is about the mass of the MBH, i.e., $M\left(<r_{h}\right) \sim M_{\bullet}$. For an isotropic cluster, the number density of the field stars is given by $n_{\star}(r)=n_{0}\left(r / r_{h}\right)^{-\alpha_{\star}}$, where $r$ is the distance from the $\mathrm{MBH}$ and $\alpha_{\star}$ is the index of the density profile, $n_{0} \simeq\left(3-\alpha_{\star}\right) M_{\bullet} /\left(4 \pi m_{\star} r_{h}^{3}\right)$ is the number density at distance $r_{h}$.

We assume that the BBHs are initially placed at distance $\sim r_{h}$ from the $\mathrm{MBH}$ and migrate into the cluster due to dynamical processes (e.g., diffusion and mass segregation by two body scatterings). We assume that the distribution of the field stars is initially in an equilibrium state, and that the existence of the BBHs does not affect the distributions of the field stars, as the total number and mass of BBHs should be always much smaller than that of the field stars.

The dynamical evolutions of the BBH within $r<r_{h}$ are significantly different from the outer parts. Both the outer and inner orbits of the BBH can be affected by various dynamical processes when they migrate into the inner regions of the cluster. Denote $a_{1}, e_{1}, \Omega_{1}, I_{1}$ and $g_{1}\left(a_{2}, e_{2}, \Omega_{2}, I_{2}\right.$ and $\left.g_{2}\right)$ as the inner (outer) semimajor axis (SMA), eccentricity, ascending node, inclination and the argument of periasis of the BBH's orbit, respectively. The outer orbit of the BBH is evolved mainly by the NR and RR. If $e_{2}$ is very high, the BBH may approach to $r_{t}$, i.e., the tidal radius of the $\mathrm{MBH}$ (see 
Table 1. Notation of Some Symbols

\begin{tabular}{|c|c|c|c|}
\hline Symbol & Description & Symbol & Description \\
\hline$E, J$ & $\begin{array}{l}\text { The energy and angular momentum of the orbit } \\
\text { circling around a MBH }\end{array}$ & $M_{\bullet}$ & The mass of the central $\mathrm{MBH}$ \\
\hline$m_{A}, m_{B}$ & The masses of the two components of a $\mathrm{BBH}$ & $\sigma_{h}$ & The velocity dispersion of the galactic nucleus \\
\hline$m_{\mathrm{BBH}}$ & The total mass of a $\mathrm{BBH}$ & $r_{h}$ & The influence radius of a $\mathrm{MBH}$ \\
\hline$m_{T}$ & $\begin{array}{l}\text { The total mass of the BBH and the incoming star } \\
\text { in the binary-single encounter }\end{array}$ & $n_{\star}$ & The number density of field stars \\
\hline$a_{1}, e_{1}$ & $\begin{array}{l}\text { The SMA and eccentricity of the inner orbit of a } \\
\text { BBH }\end{array}$ & $r_{p}$ & The pericenter of the outer orbit of a $\mathrm{BBH}$ \\
\hline$a_{2}, e_{2}$ & $\begin{array}{l}\text { The SMA and eccentricity of the outer orbit of a } \\
\text { BBH }\end{array}$ & $r_{t}$ & The tidal radius of the $\mathrm{BBH}$ around a $\mathrm{MBH}$ \\
\hline$P_{1}, P_{2}$ & $\begin{array}{l}\text { The orbital period of the inner and outer orbits of } \\
\text { a BBH }\end{array}$ & $g_{1}, g_{2}$ & $\begin{array}{l}\text { The argument of periapsis of the inner and outer } \\
\text { orbits of a BBH }\end{array}$ \\
\hline$\Omega_{1}, \Omega_{2}$ & $\begin{array}{l}\text { The ascending node of the inner and outer orbits } \\
\text { of a BBH }\end{array}$ & $I_{1}, I_{2}$ & $\begin{array}{l}\text { The orbital inclination of the inner and outer or- } \\
\text { bits of a BBH }\end{array}$ \\
\hline$\alpha_{\mathrm{BBH}}$ & $\begin{array}{l}\text { The power law index of the density profile of } \\
\text { BBHs }\end{array}$ & $\alpha_{\star}$ & $\begin{array}{l}\text { The power law index of the density profile of field } \\
\text { stars }\end{array}$ \\
\hline$x$ & $\begin{array}{l}x=E / E_{0}, \text { the normalized energy of the orbit } \\
\text { circling around a MBH. Here } E_{0}=-G M_{\bullet} / r_{h}\end{array}$ & $v_{c}$ & $\begin{array}{l}\text { Critical velocity that defines the hard and soft bi- } \\
\text { nary regions of binary-single encounters }\end{array}$ \\
\hline$g_{\mathrm{BBH}}(x)$ & $\begin{array}{l}\text { Dimensionless distribution function of the energy } \\
\text { of the BBHs' outer orbit }\end{array}$ & $g_{\star}(x)$ & $\begin{array}{l}\text { Dimensionless distribution function of the field } \\
\text { stars' orbital energy }\end{array}$ \\
\hline$m_{\star}$ & The mass of field stars & $p, b$ & $\begin{array}{l}\text { The closest distance and the impact parameter } \\
\text { between a BBH and an incoming star in a binary- } \\
\text { single encounter event }\end{array}$ \\
\hline$T_{\mathrm{GR}}$ & $\begin{array}{l}\text { The GR procession timescale of the inner orbit of } \\
\text { a BBH }\end{array}$ & $T_{\mathrm{GW}}$ & $\begin{array}{l}\text { The GW orbital decay timescale of the inner orbit } \\
\text { of a } \mathrm{BBH}\end{array}$ \\
\hline $\mathscr{R}$ & The normalized GW event rate & $e_{10 \mathrm{~Hz}}$ & $\begin{array}{l}\text { The eccentricity of the } \mathrm{BBH} \text { when its peak fre- } \\
\text { quency of GW radiation reaches to } 10 \mathrm{~Hz}\end{array}$ \\
\hline
\end{tabular}
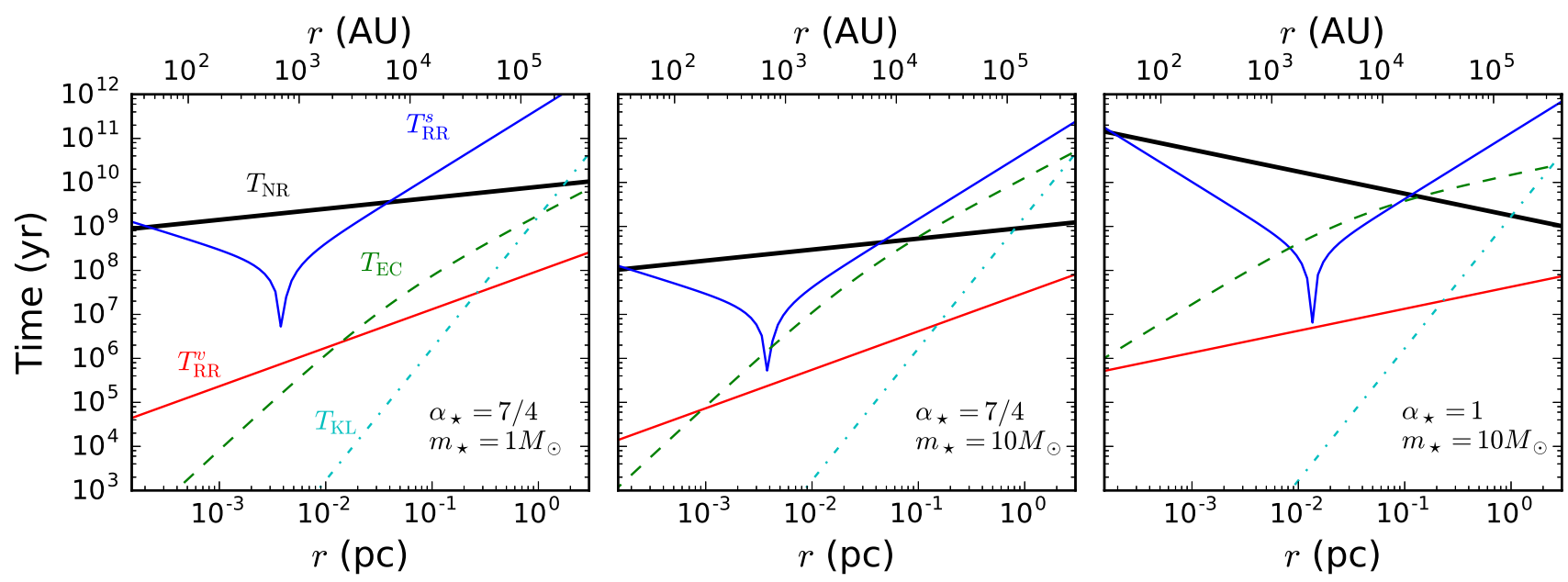

Figure 1. Timescales of different dynamical processes around a $\mathrm{MBH}$ with mass $M_{\bullet}=4 \times 10^{6} M_{\odot}$. The mass of the binary is given by $m_{A}=m_{B}=10 M_{\odot} . r$ is the distance from the $\mathrm{MBH}$. We assume $e_{2}=0.6$ for the eccentricity of the outer orbit. $T_{\mathrm{NR}}$, $T_{\mathrm{RR}}^{s}$ and $T_{\mathrm{RR}}^{v}$ are the two body relaxation timescale, scalar RR timescale and vector RR timescale, respectively. $T_{\mathrm{EC}}=R_{\mathrm{EC}}^{-1}$ is the time required to have a collision between a $\mathrm{BBH}$ and a background star, here $R_{\mathrm{EC}}$ is given by Equation C20. In all panels we have set $p=3 a_{1}$. $T_{\mathrm{KL}}$ is the KL oscillation period for a BBH-MBH triple system. Different panels show the timescales when $\alpha_{\star}$ (the density profile of the field stars) and $m_{\star}$ (the mass of the background stars) are different, as labeled in the panels. 
Eq. 5), such that its inner orbit is strongly perturbed, or likely to be disrupted after the encounter with the $\mathrm{MBH}$. It is possible that the BBHs experience multiple encounters with the field stars, which can lead to cumulative modifications of $a_{1}$ and $e_{1}$, exchange of the binary components with the incoming stars, ionization of the BBHs, or merging of the BBHs by the $\mathrm{GW}$ orbital decay. When the $\mathrm{BBH}$ is slightly away from the tidal radius, its $e_{1}$ may be excited to a high value by $\mathrm{KL}$ oscillation, such that the BBH quickly merges and triggers a GW event. During all these processes, the orbital orientations (described by $\Omega_{1}, I_{1}$ or $\Omega_{2}, I_{2}$ ) or other elements (e.g., $g_{1}, g_{2}$ ) of both the inner and outer orbits of $\mathrm{BBHs}$ can be cumulatively changed, affecting the evolutions and the merging of individual BBHs. As all of these processes may be important for the understandings of the GW merging events due to $\mathrm{BBHs}$ in galactic nuclei, here, our numerical method considers all of the above dynamical processes. The dynamical timescales for these processes for a Milky Way-like galaxy can be found in Figure 1. The details of the processes are described in the following subsections.

\subsection{Dynamics of the outer orbit}

Denote $E=-G M_{\bullet} / a_{2}$ and $J=\sqrt{G M_{\bullet} a_{2}\left(1-e_{2}^{2}\right)}$ as the energy and angular momentum of the outer orbit of a BBH. Both $E$ and $J$ are evolved under the NR. $J$ of the $\mathrm{BBH}$ is additionally affected by RR. The NR is due to the weak gravitational interactions between a $\mathrm{BBH}$ and field stars, where we treat the $\mathrm{BBH}$ as a point mass particle. The NR (or two-body relaxation) timescale is given by (Binney \& Tremaine 1987)

$$
T_{\mathrm{NR}}=\frac{0.34 \sigma^{3}}{\left(G m_{\star}\right)^{2} n_{\star} \ln \Lambda},
$$

where $\Lambda \simeq M_{\bullet} / m_{\star}$ (Bahcall \& Wolf 1976). Here $\sigma \propto$ $r^{-1 / 2}$, thus $T_{\mathrm{NR}} \propto r^{\alpha_{\star}-3 / 2}$. The time evolutions of the diffusion process in the $E-J$ space due to two-body relaxation can be described by the Fokker-Planck equation, and simulated by a Monte-Carlo scheme according to their orbit-averaged diffusion coefficients, i.e., $D_{E E}$, $D_{E}, D_{J J}, D_{J}$ and $D_{E J} \quad$ (Lightman \& Shapiro 1977). Here $D_{E E}\left(D_{J J}\right)$ and $D_{E}\left(D_{J}\right)$ describe the orbitaveraged scatterings of the energy (angular momentum) and its drift, respectively. $D_{E J}$ describes the correlations between the scatterings of energy and angular momentum. The details of the formalisms are given in Appendix A.1. We adopt a Monte-Carlo scheme similar to Shapiro \& Marchant (1978) and Bar-Or \& Alexander (2016) to calculate the two-body relaxation in $E-J$ of $\mathrm{BBHs}$ due to the field stars. More details will be explained in Section 2.5.

In the inner part of the cluster (but not too close to the $\mathrm{MBH}$, so the general relativistic precession is still not significant), RR becomes important, and the resonant torques between the orbits of the field stars can change quickly the orbital angular momentum of the BBHs. The RR changes both the amplitude (scalar RR) and the direction of the angular momentum (vector $R R$ ). The timescale of scalar $R R$ is given by (Hopman \& Alexander 2006)

$$
T_{\mathrm{RR}}^{s}=\frac{A_{\mathrm{RR}}^{s}}{t_{\omega}}\left(\frac{M_{\bullet}}{m_{\star}}\right)^{2} \frac{P^{2}\left(a_{2}\right)}{N_{\star}\left(<a_{2}\right)},
$$

where $A_{\mathrm{RR}}^{s} \simeq 3.56$ (Rauch \& Tremaine 1996). $t_{\omega}=$ $2 \pi / \nu_{p}$ is the timescale of orbital precession and $\nu_{p}$ is given by Equation A5. $P$ is the orbital period and $N_{\star}\left(<a_{2}\right)$ is the number of field stars within distance of $a_{2}$. The scalar RR can be suppressed due to the rapid general relativistic orbital precession below the locus called "Schwarzschild barrier" (SB) (Merritt et al. 2011; Antonini \& Merritt 2013), where the orbital precession frequency equals to the coherence frequency, i.e., $\nu_{p}=2 \pi / T_{c}\left(a_{2}\right)$ (Bar-Or \& Alexander 2016). Here $T_{c}\left(a_{2}\right)$ is the coherence timescale given by Equation A9.

The timescale of vector $R R$ is given by (Hopman \& Alexander 2006)

$$
T_{\mathrm{RR}}^{v}=2 A_{\mathrm{RR}}^{v}\left(\frac{M_{\bullet}}{m_{\star}}\right) \frac{P\left(a_{2}\right)}{N_{\star}^{1 / 2}\left(<a_{2}\right)},
$$

where $A_{\mathrm{RR}}^{v} \simeq 0.31$ (Rauch \& Tremaine 1996). By a method similar to Bar-Or \& Alexander (2016), we can consider the scalar RR by calculating the diffusion coefficients in angular momentum $D_{J J}^{R R}$ and $D_{J}^{R R}$. For vector $R R$, we simply consider it as a diffusion process. The details of the scalar and vector $R R$ are shown in Appendix A.2.

\subsection{Tidal disruption of $B B H s$}

During the evolution, if the $\mathrm{BBH}$ approaches too close to the $\mathrm{MBH}$, they will likely be disrupted by the tidal force of the MBH. The tidal radius of binary stars is given by

$$
r_{t}=\left(\frac{3 M_{\bullet}}{m_{\mathrm{BBH}}}\right)^{1 / 3} a_{1},
$$

where $m_{\mathrm{BBH}}=m_{A}+m_{B}$ is the total mass of the binary. The loss cone of the angular momentum is $J_{\mathrm{lc}}^{2} \simeq 2 M_{\bullet} r_{t}$. The empty loss cone region requires that the change of angular momentum per orbital period of the BBH is smaller than the size of lose cone, i.e., $d J<J_{\text {lc }}$ and the full loss cone region requires that $d J>J_{\text {lc }}$. In full lose cone region, the binary can jump in and out of the lose cone multiple times before it encounters with the MBH. In empty loss cone region, the binary takes multiple periods to move into the lose cone and may have encountered with the MBH multiple times. As the probability of 
tidal disruption is a function of distance from the $\mathrm{MBH}$ (e.g., in the case of binary stars (Zhang et al. 2010)). The BBH may experience multiple encounters with the $\mathrm{MBH}$ before the tidal disruption. There are also chances that the $\mathrm{BBH}$ moves away from the lose cone after the multiple encounters with the MBH. Zhang et al. (2010) have showed that the multiple encounters can change both the SMA and the eccentricity of the binary, which thus may lead to merging events.

To consider accurately such effects, we use three-body numerical integrations to calculate the outcome of each encounters between the $\mathrm{BBH}$ and the $\mathrm{MBH}$. Considering that the $\mathrm{MBH}$ can impose strong impacts on the inner orbit of the $\mathrm{BBH}$ even before it reaches the loss cone, we set up the three body integration when $a_{2}\left(1-e_{2}\right)<3 r_{t}$. At the beginning of these explicit three-body simulations, initially the binary is placed at $10^{4} a_{1}$ (or at apocenter of the outer orbit if $a_{2}$ is smaller). After the encounter, the change of the inner orbit of the binary at distance $10^{4} a_{1}$ from the MBH is recorded for next encounters. Such a process repeats till either the binary is disrupted, or merged due to the GW emission, or moving out of the loss cone.

\subsection{Kozai-Lidov Effect and the Gravitational wave orbital decay}

The BBH-MBH system forms a natural triple system and the tidal force of the MBH can trigger KL oscillation on the inner and outer orbits of the BBH. The KL effect will increase the eccentricity of the inner orbit of the binary under some preferred orbital configurations (e.g. Naoz et al. 2013; Naoz 2016). The period of KL oscillation is given by (Kiseleva et al. 1998)

$$
\begin{aligned}
& T_{K}=\frac{2 P_{2}^{2}}{3 \pi P_{1}}\left(1-e_{2}^{2}\right)^{3 / 2} \frac{m_{A}+m_{B}+M_{\bullet}}{M_{\bullet}} \\
& \simeq \frac{2}{3^{1 / 3} \pi}\left(\frac{r_{p}}{r_{t}}\right)^{3 / 2} P_{2} .
\end{aligned}
$$

The GR procession of the inner orbit of the binary is given by

$$
T_{\mathrm{GR}}=\frac{2 \pi a_{1}^{5 / 2} c^{2}\left(1-e_{1}^{2}\right)}{3 G^{3 / 2}\left(m_{A}+m_{B}\right)^{3 / 2}} .
$$

If $T_{\mathrm{GR}}<T_{K}$, or,

$$
r_{p} \lesssim 0.58\left(\frac{T_{\mathrm{GR}}}{P_{2}}\right)^{2 / 3} r_{t},
$$

the KL effect will be suppressed (e.g., Ford et al. 2000; Naoz et al. 2013). In most cases, the KL effect is only significant when the pericenter of the outer orbit of the $\mathrm{BBH}$ is close to the tidal radius of the MBH. In this work, we consider the KL effect only when $r_{p}$ falls in the range of $3 r_{t}$ to $\min \left[20,0.58\left(T_{\mathrm{GR}} / P_{2}\right)^{2 / 3}\right] r_{t}$. When $r_{p}<3 r_{t}$ we use the explicit three-body integration to calculate the interactions between the BBHs and the MBH (See Section 2.2).

When the eccentricity of the inner orbit, $e_{1}$, is excited to a high value due to KL effect (or other effects), the $\mathrm{GW}$ radiation becomes important and the $\mathrm{BBH}$ is likely to be merged in a very short timescale. The GW orbital decay timescale is given by (Peters 1964)

$$
T_{\mathrm{GW}}=\frac{5}{64} \frac{c^{5} a^{4}}{m_{A} m_{B}\left(m_{A}+m_{B}\right) G^{3}} \frac{\left(1-e_{1}^{2}\right)^{7 / 2}}{1+\frac{73}{24} e_{1}^{2}+\frac{37}{96} e_{1}^{4}} .
$$

The evolution of the SMA and eccentricity of the inner binary by GW radiation is given by (Peters 1964)

$$
\begin{aligned}
& \dot{a}_{1}^{\mathrm{GW}}=-\frac{64}{5} \frac{m_{A} m_{B}\left(m_{A}+m_{B}\right) G^{3}}{c^{5} a_{1}^{3}} \frac{1+\frac{73}{24} e_{1}^{2}+\frac{37}{96} e_{1}^{4}}{\left(1-e_{1}^{2}\right)^{7 / 2}}, \\
& \dot{e}_{1}^{\mathrm{GW}}=-\frac{304}{15} e_{1} \frac{m_{A} m_{B}\left(m_{A}+m_{B}\right) G^{3}}{c^{5} a_{1}^{4}} \frac{1+\frac{121}{304} e_{1}^{2}}{\left(1-e_{1}^{2}\right)^{5 / 2}} .
\end{aligned}
$$

Here the dot means the derivative respect to time $t$. When the eccentricity is high, the argument of pericenter (denoted as $g_{1}$ ) of the BBH's inner orbit can precess significantly. For simplicity, we consider only the postNewtonian GR effect, i.e.,

$$
\dot{g}_{1}^{\mathrm{PN}}=\frac{3\left(m_{A}+m_{B}\right)^{3 / 2} G^{3 / 2}}{c^{2} a_{1}^{5 / 2}\left(1-e_{1}^{2}\right)} .
$$

We consider the evolution of the inner and outer orbits of the BBHs under the above two effects and the KL oscillations. For KL effect, we adopt the time evolution formalism in Naoz et al. (2013). The equations of motion in $a_{1}, e_{1}$ and $g_{1}$ now become

$$
\dot{a}_{1}=\dot{a}_{1}^{\mathrm{GW}}, \quad \dot{e}_{1}=\dot{e}_{1}^{\mathrm{GW}}+\dot{e}_{1}^{\mathrm{KL}}, \quad \dot{g}_{1}=\dot{g}_{1}^{\mathrm{PN}}+\dot{g}_{1}^{\mathrm{KL}} .
$$

Here $\dot{e}_{1}^{\mathrm{KL}}, \dot{g}_{1}^{\mathrm{KL}}$ can be found in Naoz et al. (2013). For the KL evolution of the other orbital elements of BBHs, see Naoz et al. $(2013)^{1}$. Recent studies show that these analytical formula capture only the secular terms of the evolution, while the non-secular terms may be important for a BBH-MBH triple system (Grishin et al. 2018). The non-secular terms can enhance the maximum eccentricity in oscillations and increase the merging rates by up to an order of magnitude (Fragione et al. 2018). Thus, our merging rates due to KL effect may have been underestimated.

It would be interesting to investigate the eccentricity and the SMA when the peak of GW frequency enters to

\footnotetext{
1 We have noticed that Naoz et al. (2013) does not consider the gravitational radiation loss of the total angular momentum $H$ (see Blaes et al. (2002)). Nevertheless, it should not affect much of our simulation results, as such radiation loss on $H$ should be only important when the gravitational wave orbital decay dominates the motion, and could be ignored during most time of the KL oscillation.
} 

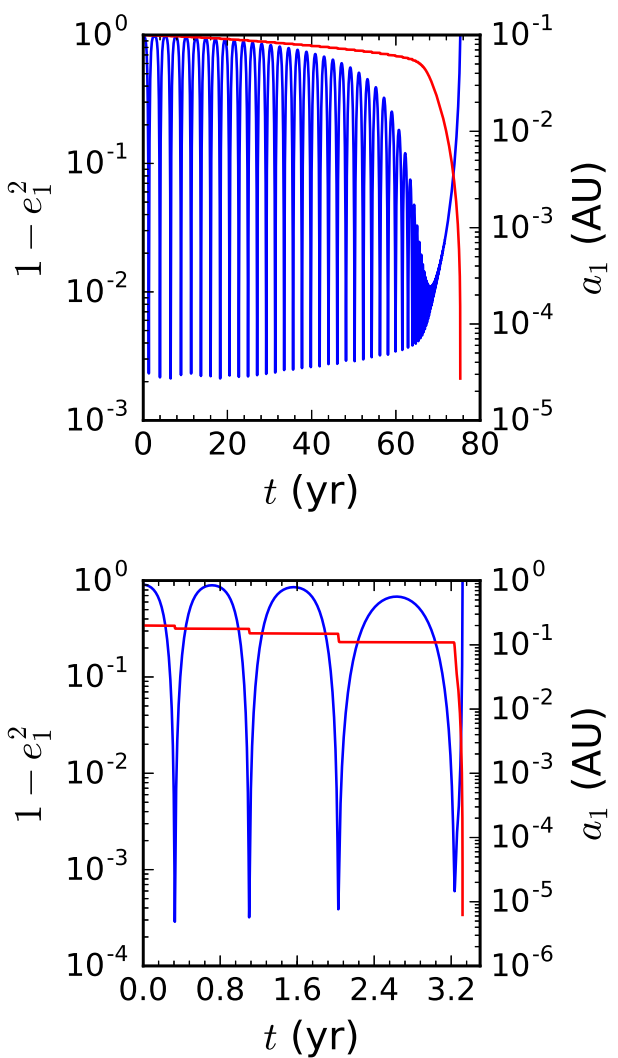
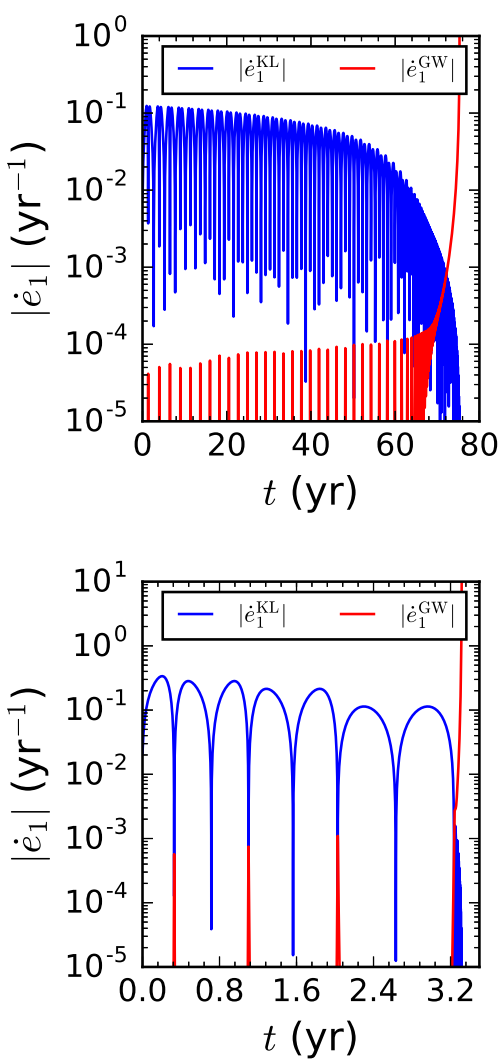
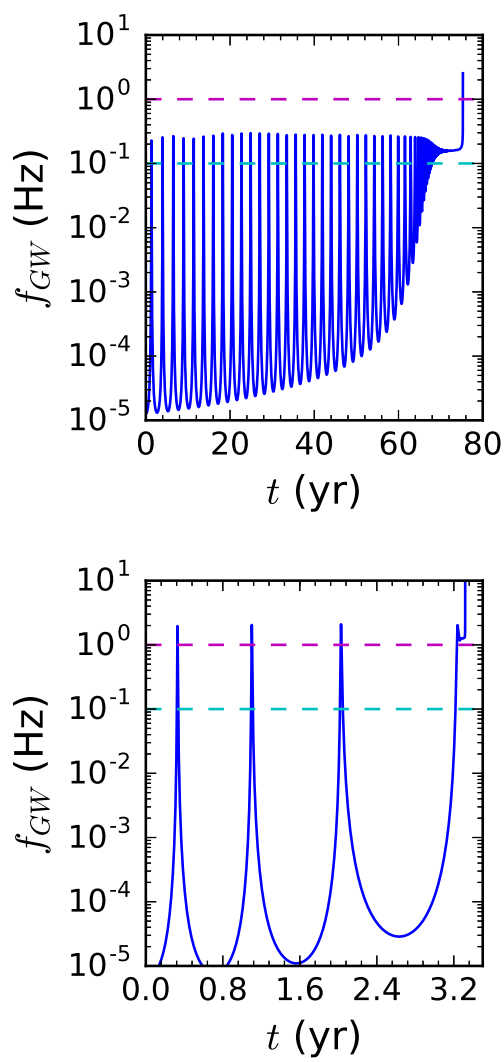

Figure 2. Left panels: The time evolution of $1-e_{1}^{2}$ (solid blue lines) and $a_{1}$ (solid red lines) of the BBHs before they merge. Middle panels: The time evolution of $\left|\dot{e}_{1}^{\mathrm{GW}}\right|$ (solid blue lines) and $\left|\dot{e}_{1}^{\mathrm{KL}}\right|$ (solid red lines), which are given by Equation 10 and from Naoz et al. (2013), respectively. Right panels: The time evolution of the peak of GW frequency, given by Equation B11. The magenta dashed line shows $f_{\mathrm{GW}}=1 \mathrm{~Hz}$, above which the GW can be observed by the ground-based GW detectors. The cyan dashed line shows $f_{\mathrm{GW}}=0.1 \mathrm{~Hz}$. The upper panels are results from a BBH assuming $a_{1}=0.1 \mathrm{AU}, e_{1}=0.1, m_{A}=10 M_{\odot}$, $m_{B}=20 M_{\odot}$. The peak frequency $f_{\mathrm{GW}}$ is larger than $1 \mathrm{~Hz}$ after the GW dominates the evolution of the BBH. For bottom panels, the assumed $\mathrm{BBH}$ is similar but with $a_{1}=0.2 \mathrm{AU}$ and $e_{1}=0.3$. Different from the upper panels, the peak frequency is larger than $1 \mathrm{~Hz}$ before the $\mathrm{GW}$ dominates the evolution of the $\mathrm{BBH}$. For all panels the outer orbit of the $\mathrm{BBH}$ is given by $a_{2}=50 \mathrm{AU}$ and $e_{2}=0.3$.

the observing band of ground-based GW detectors, i.e., $f_{\mathrm{GW}} \gtrsim 1 \mathrm{~Hz}$. We discribe the method in Appendix B, that mainly bases on the Equations B11 and B12. Figure 2 illustrates the two possible cases of merging a $\mathrm{BBH}$. The first one is shown by the top panels, i.e., when the GW frequency approaches to $f_{\mathrm{GW}}=1 \mathrm{~Hz}$, the $\mathrm{GW}$ dominates the evolution of the $\mathrm{BBH}$ and the eccentricity of the $\mathrm{BBH}$ reduces to $e_{1} \sim 0.1$. Alternatively, as the bottom panels indicate, $f_{\mathrm{GW}}$ can be larger than 1 $\mathrm{Hz}$ before the GW dominates, and the eccentricity of the $\mathrm{BBH}$ is extremely high, i.e., $1-e_{1} \sim 10^{-3}-10^{-4}$. We find that the latter case is very rare, i.e., $<0.1 \%$ of all the $\mathrm{BBH}$ mergers in our simulation. However, if the entering frequency can be as low as about $\sim 0.1 \mathrm{~Hz}$, then the probability can be much higher; the inspiraling phase of BBHs, before GW dominating the evolution, can now be observable (see top right panel of Figure 2). If such phenomena can be detected, probably in decihertz GW detectors (Mandel et al. 2018), it can be a strong evidence that those GW mergers are due to $\mathrm{KL}$ oscillations.

\subsection{Binary-single encounters}

The BBH will experience encounters with the field stars (or stellar remnants) if the stellar density is high. Such encounters can change both $a_{1}$ and $e_{1}$, or even lead to exchange of the binary components. There is a critical velocity that defines the hard and soft regions of the binary encounter (Hut \& Bahcall 1983),

$$
v_{c}=\sqrt{\frac{G m_{T}}{a_{1}} \frac{\mu_{12}}{m_{3}}},
$$

where $\mu_{12}=m_{A} m_{B} /\left(m_{A}+m_{B}\right)$ is the reduced mass of the binary, $m_{T}=m_{A}+m_{B}+m_{3}$ is the total mass, and $m_{3}$ is the mass of the incoming star. Suppose that the velocity of the incoming star with respect to the mass center of BBHs is $v_{\infty}$, if $v_{\infty}>v_{c}$, the binary is soft, and is hard if $v_{\infty}<v_{c}$. After the encounter, a hard binary 

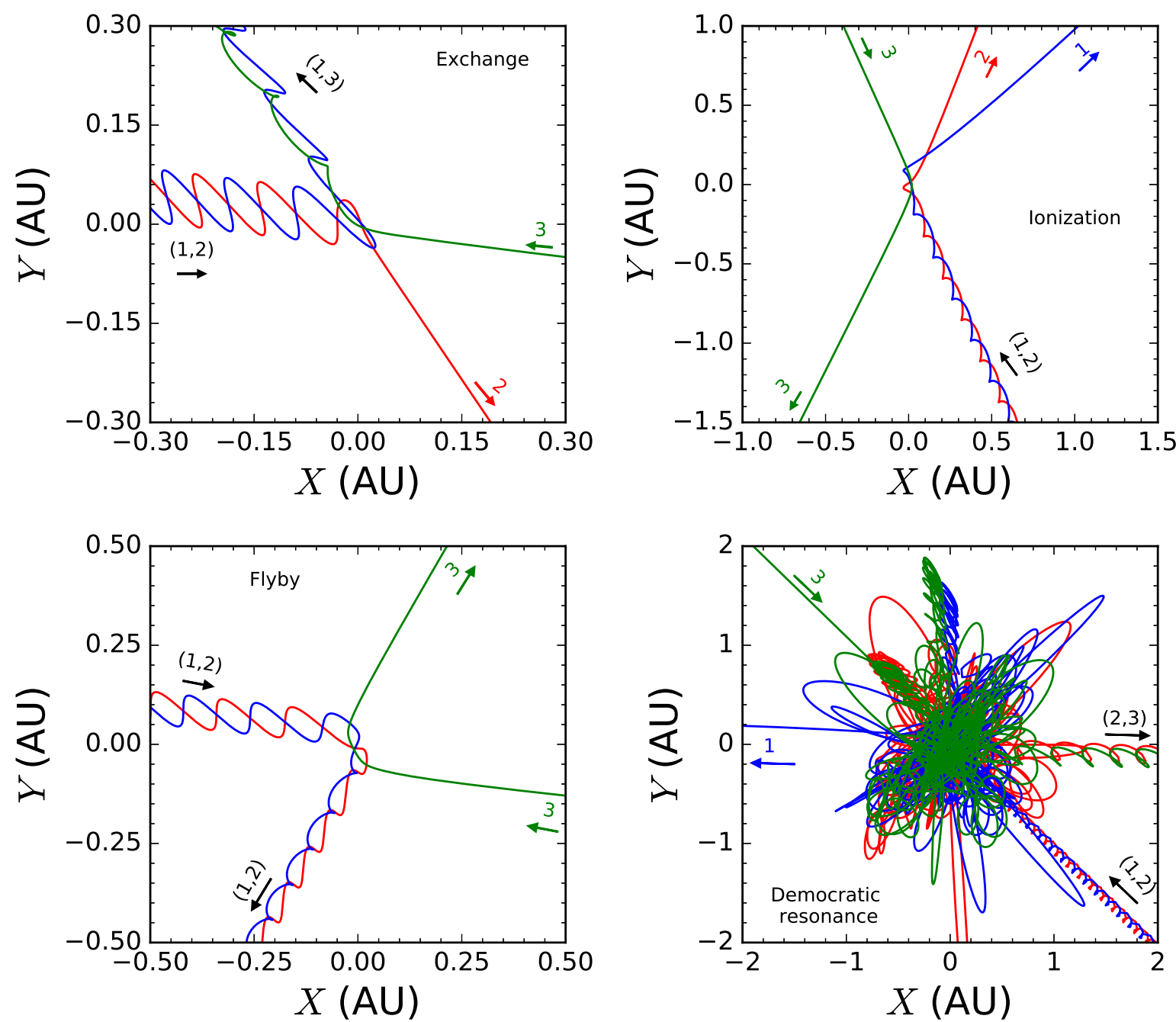

Figure 3. Four possible outcomes of an encounter between a BBH and a background star. All panels show the trajectory of the $\mathrm{BBH}$ and the incoming star in the projected $X$ and $Y$ axis. The number 1 and 2 refer to the two components of the binary and 3 refer to the incoming field star. In all panels the initial SMA of the BBH is $a_{1}=0.1 \mathrm{AU}$.

always becomes harder, while for a soft binary, it could become harder or softer, or even ionized (disrupted).

The event rates of binary-single encounter depend on the density profiles, whether the binary is hard or soft, and other details of the cluster. The detailed calculations can be found in Appendix C. Assuming that $\alpha_{\star}=1.75$, for a soft binary, the event rate is given by

$$
\begin{aligned}
R_{\mathrm{EC}} & =2.23 \times 10^{-7} \mathrm{yr}^{-1} \Theta\left(\frac{p}{0.1 \mathrm{AU}}\right)^{2}\left(\frac{a_{2}}{10^{3} \mathrm{AU}}\right)^{-9 / 4} \\
& \times\left(\frac{M_{\bullet}}{4 \times 10^{6} M_{\odot}}\right)^{7 / 8}\left(\frac{10 M_{\odot}}{m_{\star}}\right)
\end{aligned}
$$

and for a hard binary, we have

$$
\begin{aligned}
R_{\mathrm{EC}} & =1.88 \times 10^{-9} \mathrm{yr}^{-1} \Phi\left(\frac{p}{0.1 \mathrm{AU}}\right)\left(\frac{a_{2}}{10^{4} \mathrm{AU}}\right)^{-5 / 4} \\
& \times\left(\frac{M_{\bullet}}{4 \times 10^{6} M_{\odot}}\right)^{-1 / 8}\left(\frac{10 M_{\odot}}{m_{\star}}\right)\left(\frac{m_{T}}{30 M_{\odot}}\right),
\end{aligned}
$$

where $\Theta$ and $\Phi$ are dimensionless constants of order unity, which depends slightly on the orbital eccentricity $e_{2}$ (See Appendix C for more details). $p$ is the pericenter distance of the incoming star with respect to the $\mathrm{BBH}$, and it relates to the impact parameter $b$ by

$$
b=p \sqrt{1+2 m_{T} G /\left(v_{\infty}^{2} p\right)} .
$$

We can see that, for a typical galactic nucleus, the binary-single encounter should be considered in the simulation, as they could be frequent, especially in the inner regions of the nucleus cluster (see also Figure 1). 
As the outcome of a binary-single encounter is complex and we find that the change of the inner orbit of the $\mathrm{BBH}$ may have significant impacts on their GW merging rates, such a process should be calculated as accurately as possible. Here we use explicit three-body numerical integration to calculate the outcome of the binary-single encounters. The incoming star is assumed coming from infinity with $v_{\infty}=\sqrt{G M_{\bullet} /\left(2 a_{2}\right)}$ and impact parameter $0<b<b_{\max }$ which follows a distribution $f(b) \propto b$. Here $b_{\max }$ relates to $p_{\max }$ according to Equation 16 . We set $p_{\max }=\max \left[6\left(m_{\star} / m_{\mathrm{BBH}}\right)^{1 / 2}, 2\right] a_{1}\left(1+e_{1} / 2\right)$ such that the simulation results can be converged. As $m_{\star} / m_{\mathrm{BBH}} \sim 1-0.1, p_{\max } \sim 2 a_{1}-9 a_{1}$. The initial orbital orientation of the incoming star is set to be random. Initially the incoming star is put at distance of $50 a_{1}$ away from the BBH. After each encounter, we determine the outcomes according to the relative energies and separations between each particle (black holes or incoming stars).

Figure 3 illustrates the four most likely outcomes of binary-single encounters in our simulation: (1) Exchange of one of the binary components with the incoming object. This event is common if $m_{\mathrm{BBH}} \lesssim m_{\star}$, i.e., when the incoming object is massive. The incoming object is most likely a star considering that the number fraction of black holes is very small $\left(\lesssim 10^{-2}\right.$, see Hopman \& Alexander (2006)), thus, the exchange event usually leads to a non-BBH object. In the current work, we simply remove such binaries in the simulation. However, we notice that in the future our work can be easily expanded to further consider the evolution of binaries of which one component being a star or a neutron star and the other being a black hole. (2) Ionization, which means that the binary is destroyed due to the encounter. This event is only possible if $v_{\infty}>v_{c}$, i.e., when the binary is soft. (3) Flyby, the binary remains integrity after the encounter. However, the energy and angular momentum of the inner binary, or equivalently, $a_{1}$ and $e_{1}$, are both changed. (4) Three bodies experience chaotic evolutions, and finally, a binary is formed and the third star being ejected from the system. These events are only possible if $v_{3}<v_{c}$, i.e., when the binary is hard. There are other possible outcomes, e.g., hierarchical resonants (Heggie \& Hut 1993), however, only for hard binaries. As most of the binaries in our work are soft binaries, we do not discuss more details of them here.

\subsection{The Monte-Carlo Schemes}

We use the Monte-Carlo Schemes that are similar to Shapiro \& Marchant (1978) to simulate the evolution of the inner and outer orbits of a $\mathrm{BBH}$ around the $\mathrm{MBH}$. Our method has combined all of the effects described above, i.e., the NR and RR of the BBHs (Sec- tion 2.1), the tidal disruption of the BBHs (Section 2.2), the $\mathrm{KL}$ oscillations and the GW radiation of the inner orbit of the BBHs (Section 2.3), and the binary-single encounters (Section 2.4). The details of the method are described as follows.

Define $x=E / E_{0}$ as the dimensionless energy, where $E_{0}=-G M_{\bullet} / r_{\mathrm{h}}$ is the characteristic energy. We assume that the energy distribution of the field stars is given by (Lightman \& Shapiro 1977; Magorrian \& Tremaine 1999) $f(E)=\left(2 \pi \sigma_{0}^{2}\right)^{-3 / 2} n_{0} g_{\star}(x)$, where

$$
g_{\star}(x)=\frac{\Gamma\left(\alpha_{\star}+1\right)}{\Gamma\left(\alpha_{\star}-1 / 2\right)} x^{\alpha_{\star}-3 / 2}, \quad 3>\alpha_{\star}>1 / 2,
$$

and $x>0$, where $\alpha_{\star}$ is the power law index of density profile of the field stars. Here $\sigma_{h}^{2}=G M_{\bullet} / r_{h}, n_{0}$ is the number density of the stars at position $r=r_{h}$. If $x<0$, we simply set $g_{\star}(x)=e^{x}$ (Lightman \& Shapiro 1977).

We initially put all the BBHs near the edge of the influence radius of the $\mathrm{MBH}$, i.e., $r=r_{\mathrm{i}}=r_{\mathrm{h}}($ or $x=0.5)$. The initialization of the inner orbits of the binaries depends on the problems concerned, and will be introduced in more detail at the beginning of Section 3 and Section 4. The dimensionless distribution function of the energy of the BBHs' outer orbit is defined by:

$$
g_{\mathrm{BBH}}(x)=\frac{n(x)}{n(1)} \frac{\Gamma\left(\alpha_{\star}+1\right)}{\Gamma\left(\alpha_{\star}-1 / 2\right)} x^{5 / 2},
$$

where $n(x)$ is the number distribution of BBHs. This is obtained according to $n(E) \propto f(E) E^{-5 / 2}$ in an isotropic cluster, where $n(E)$ is the number density of BBHs as a funtion of the outer orbits' energy (Hopman 2009). In the above equation, $g_{\mathrm{BBH}}$ is normalized for the convenience of model comparison, as $g_{\mathrm{BBH}}(1)=g_{\star}(1)$ for all models with the same $\alpha_{\star}$.

If $t$ is the current time of the simulation, we take the following steps for each $\mathrm{BBH}$ :

1. A time step size $\delta t$ is determined according to Section 2.6.

2. We trace the NR of the energy $\left(E=-G M_{\bullet} /\left(2 a_{2}\right)\right)$ and $\mathrm{NR}$ and $\mathrm{RR}$ of the angular momentum $(J=$ $\left.\sqrt{G M_{\bullet} a_{2}\left(1-e_{2}^{2}\right)}\right)$ of the outer binary by the method described in Section 2.1. The change of energy and angular momentum is given by

$$
\begin{aligned}
d E= & D_{E}^{N R} \delta t+y_{1} \sqrt{D_{E E}^{N R} \delta t}, \\
d J= & \left(D_{J}^{N R}+D_{J}^{R R}\right) \delta t, \\
& +y_{2} \sqrt{D_{J J}^{N R} \delta t}+y_{3} \sqrt{D_{J J}^{R R} \delta t .}
\end{aligned}
$$

Here $y_{1}, y_{2}$ and $y_{3}$ are unity normal random variables. Note that $y_{1}$ and $y_{2}$ have correlations $\rho=D_{E J} / \sqrt{\left|D_{E E} D_{J J}\right|}$. They can be obtained by generating a bi-normal distribution and then taking the transformation, $y_{2} \rightarrow y_{1} \rho+y_{2} \sqrt{1-\rho^{2}}$. 
3. If the pericenter of the outer orbit of a $\mathrm{BBH}$ is within $r_{p}<3 r_{t}$, and when $\operatorname{int}(t / P)-\operatorname{int}[(t-$ $\delta t) / P]>0$, i.e., this $\mathrm{BBH}$ is near loss cone for an orbital period, we start a three body simulation, that traces the interactions between this $\mathrm{BBH}$ and the MBH (see Section 2.2 for more details).

4. The expected number of collisions between each $\mathrm{BBH}$ with the field stars is calculated according to $k=R_{\mathrm{EC}} \delta t$, where $R_{\mathrm{EC}}$ is given by Equation C20. The realized number of collisions, $n_{\mathrm{EC}}$, for each $\mathrm{BBH}$ is generated according to the Poisson distribution $P_{\mathrm{EC}}(k)$ (given by Equation C21). We then take a number of $n_{\mathrm{EC}}$ successive three body integration to consider these encounter events (the details can be found in Section 2.4). Usually, the timestep is set such that $n_{\mathrm{EC}} \sim 1$ (see Section 2.6). Note that any change of the inner orbit of the BBH is saved for next steps. After each encounter, the $\mathrm{BBH}$ is considered to be ionized if $\epsilon<10^{-3} m_{\star}|E|$, here $\epsilon=m_{\mathrm{BBH}}^{2} /\left(2 a_{1}\right)$.

5. If the pericenter of the outer orbit of a $\mathrm{BBH}$ is $3 r_{t}<r_{p}<\min \left[20,0.58\left(T_{\mathrm{GR}} / P_{2}\right)^{2 / 3}\right] r_{t}$, we consider the KL oscillation of the inner binary that lasts for a time period of $\delta t$ (see Section 2.3 for more details). We calculate the $\mathrm{GW}$ radiation timescale $T_{\mathrm{GW}}$ for this $\mathrm{BBH}$ at any moment, and the $\mathrm{BBH}$ is considered to be merged due to GW radiation if $\delta t-t^{\prime}<50 T_{\mathrm{GW}}$, where $0<t^{\prime}<\delta t$ is the current time of simulation in the KL subroutines. We calculate the eccentricity and SMA of the BBH when it reaches $f_{\mathrm{GW}}=10 \mathrm{~Hz}$ according to Appendix B. If $\left|\dot{e}_{1}^{\mathrm{KL}}\right|>\left|\dot{e}_{1}^{\mathrm{GW}}\right|$ and $f_{\mathrm{GW}} \geq 10 \mathrm{~Hz}$, the $\mathrm{BBH}$ is now entering the LIGO frequency band, and we record the current value of eccentricity and SMA of the BBH.

6. If $r_{p}>\min \left[20,0.58\left(T_{\mathrm{GR}} / P_{2}\right)^{2 / 3}\right] r_{t}$, we calculate the GW radiation of the inner binary according to Equation 10. Similarly, we consider the BBH is merged if $10 T_{\mathrm{GW}}<\delta t$, and we calculate the eccentricity and SMA of the BBH when it reaches $f_{\mathrm{GW}}=10 \mathrm{~Hz}$ according to Appendix B.

7. We remove any BBH that has a dimensionless energy of $x<0.1$ or $x>10^{4}$. The inner boundary corresponds to a distance of about $10 \mathrm{AU}$ if $M_{\bullet}=10^{6}-10^{7} M_{\odot}$.

8. If the $\mathrm{BBH}$ is not destroyed (due to either merger of GW radiation or ionization, or tidal disruption, etc), repeat step 1 to step 7 till the density profile of BBHs, i.e., $g_{\mathrm{BBH}}$, reach an equilibrium state ${ }^{2}$. For any $\mathrm{BBH}$ that is destroyed, its simulation ends and the information is saved for statistics. The information include the inner and outer orbits of the $\mathrm{BBH}$, the current simulation time, the number of different dynamical events it has been experienced, and etc.

We trace the evolution of the ascending node $\left(\Omega_{1}, \Omega_{2}\right)$, inclination $\left(I_{1}, I_{2}\right)$ and the argument of periapsis $\left(g_{1}, g_{2}\right)$ for both the inner and outer orbits of the BBHs. Note that all these elements are defined with respect to an arbitrary selected reference plane and a direction within it, which are the same for all BBHs in a simulation. We assume that the vector $\mathrm{RR}$ process affects only the orientation of the outer orbit $\left(\Omega_{2}\right.$ and $\left.I_{2}\right)$, but not the inner orbit. During the binary-single encounter, the orbital elements of the inner orbit are changed and recorded for successive encounters. Currently, we do not consider the change of the outer orbit due to the binary-single encounter, but defer it to future studies. Such simplification should not significantly affect our results as the velocity dispersion in the cluster is usually much larger than the velocity of the inner orbits of BBHs. All angles are considered and traced in the KL oscillations and the BBH-MBH encounters. Note that the additional precession of $g_{2}$ due to distributed mass has not been included in the current simulation (See Section 5 for how it affects our results).

As sometimes the density of the binaries drops rapidly near the $\mathrm{MBH}$, to increase the number statistics we use the "clone scheme" similar to Shapiro \& Marchant (1978). We generate a number of $\Pi-1$ clones of a BBH (either an original or a clone one) when its energy crosses the boundary $x=10^{i}$ from lower $x$, where $i=1,2,3$. Here the number $\Pi$ is the amplification factor in the scheme, and is usually a number selected between $2-10$ in each run, such that the number of BBHs in both outer and inner regions of the cluster is sufficiently large for statistics. If a clone particle crosses such boundary from higher $x$, it will be removed from the simulation (its information is not saved for statistics). When a $\mathrm{BBH}$ is destroyed because of any step in (3)-(7), if it is the original particle, a new particle (regarded also as a new original particle) at $r=r_{\mathrm{i}}$ is generated. But if it's a clone particle, it is removed from the simulation. Note

\footnotetext{
2 The equilibrium state is considered when the total time of simulation $t>T_{\mathrm{NR}}(r)$, where $T_{\mathrm{NR}}$ is given by Equation 2 . Here $r=r_{h} / 2$ if $\alpha_{\star}=7 / 4$ and $r=0.001 r_{h}$ if $\alpha_{\star}=1$. In each simulation we output the density profile $g_{\mathrm{BBH}}(x)$ at different time snapshot which usually is separated by about $\sim 0.1-0.5 T_{\mathrm{NR}}$. The simulation continues until the $g_{\mathrm{BBH}}(x)$ of the last three snapshots converge. The last snapshot is considered as the equilibrium state and used for statistics.
} 
that any $\mathrm{BBH}$ located at position $10^{i}<x<10^{i+1}$ has a statistical weight of $\Pi^{-i}$.

We use the code DORPI5 based on the explicit fifth (fourth)-order Runge Kutta method (Dormand \& Prince 1980; Hairer et al. 1993) to calculate the 3-body dynamics of the binary-single encounters and binary-MBH encounters, and the equations of the motion of KL oscillation. The level of integration accuracy is always below $10^{-12}$, which should be small enough for the convergence of the simulation results.

\subsection{Timesteps}

The timestep in our simulation should be small enough, such that the results can converge. We use the step control similar to Shapiro \& Marchant (1978). The timestep $\delta t$ satisfies

$$
\begin{aligned}
& \min \left(\delta t\left|D_{E}^{N R}\right|, \sqrt{\delta t D_{E E}^{N R}}\right) \leq 0.15|E|, \\
& \max \left(\sqrt{\delta t D_{J J}^{N R}}, \sqrt{\delta t D_{J J}^{R R}}\right) \leq \min \left[0.1 J_{\mathrm{c}}, 0.4\left(1.0075 J_{c}-J\right)\right], \\
& \max \left(\sqrt{\delta t D_{J J}^{N R}}, \sqrt{\delta t D_{J J}^{R R}}\right) \leq \max \left(0.25\left|J-J_{\mathrm{lc}}\right|, 0.1 J_{\mathrm{lc}}\right) .
\end{aligned}
$$

Also, if $r_{p}=a_{2}\left(1-e_{2}\right)<3 r_{t}$, we set $\delta t \leq P$, where $P$ is the orbital period, such that the three body encounter between the $\mathrm{BBH}$ and the $\mathrm{MBH}$ is considered for each period. Under these conditions, the step size is small enough, that the change of angular momentums is always smaller than the size of the lose cone.

Also, if the binary-single encounters are considered, we require that the time step size is small enough, that in each step the number of binary-single collisions is of order unity,

$$
\delta t \leq R_{\mathrm{CO}}^{-1}
$$

Here $R_{\mathrm{CO}}$ is given by Equation C20. If the vector relaxation is considered, to avoid a large change of the orientation of the angular momentum, we set the time step such that the change of angles is less than $30^{\circ}$, i.e.,

$$
\delta t \leq 0.25 T_{\mathrm{RR}}^{v}
$$

where $T_{\mathrm{RR}}^{v}$ is given by Equation 4 .

\section{SIMULATIONS: THE EFFECTS OF DIFFERENT DYNAMICAL PROCESSES}

In order to explore the effect on the density distribution of the BBHs and the GW merge rates by different dynamical effects, we perform simulations according to Section 2 for some simplified models. In this section we fix the $\mathrm{MBH}$ mass to be $4 \times 10^{6} M_{\odot}$ (which is the case of the Milky Way) and fix the initial SMA of the inner orbit and mass of the binary. For a more general and realistic initial condition that provides more accurate es-
Table 2. Models

\begin{tabular}{lcccccc}
\hline & $m_{\mathrm{BBH}}\left(M_{\odot}\right)$ & $m_{\star}\left(M_{\odot}\right)$ & $a_{1}(\mathrm{AU})$ & $e_{1}$ & $\alpha_{\star}$ & $\xi^{a}$ \\
\hline M1 & $5 \times 2$ & 10 & 0.1 & 0 & 1.75 & 1.5 \\
M2 & $10 \times 2$ & 1 & 0.1 & 0 & 1.75 & 59 \\
M3a & $10 \times 2$ & 10 & 0.1 & 0 & 1.75 & 5.9 \\
M3b & $10 \times 2$ & 10 & 0.1 & 0 & 1 & 7.5 \\
M3c & $10 \times 2$ & 10 & 0.1 & $\mathrm{THM}^{b}$ & 1.75 & 5.9 \\
M4 & $30 \times 2$ & 10 & 0.2 & 0 & 1.75 & 53 \\
\hline
\end{tabular}

Note- All the models have $M_{\bullet}=4 \times 10^{6} M_{\odot}$.

${ }^{a}$ The initial value of $\xi$ for the binary at $r=r_{h}$ is given by Equation 24.

${ }^{b}$ Thermal distribution, i.e., $f(e) \mathrm{d} e=2 e \mathrm{~d} e$.

timations of the total GW event rates of $\mathrm{BBH}$ mergers for all of the local galaxies, see Section 4 .

The initial conditions of some typical models are given in Table 2. We assume two cases of the density profile of the field stars, i.e., $\alpha_{\star}=7 / 4$, which corresponds to the value expected around a MBH (Bahcall \& Wolf 1976), or $\alpha_{\star}=1$, which corresponds to a core-like cluster with a shallower density profile. For the models in Table 2, initially the mass of the background particle is $m_{\star}=1 M_{\odot}$ or $10 M_{\odot}$. The former one assumes that later-type stars dominate the central regions while the latter one assumes that black holes dominate. We assume that the two components of $\mathrm{BBH}$ are of equal mass, and with mass $5 M_{\odot}, 10 M_{\odot}$ or $30 M_{\odot}$ in different models. In the case of $m_{A}=m_{B}=5 M_{\odot}$ the mass of the BBH equals to the field stars, thus the density profile of them should follows also the cusp profile, i.e., $\alpha_{\star}=7 / 4$. We consider the case of $m_{A}=m_{B}=30 M_{\odot}$ such that they are similar to the masses of the merged $\mathrm{BBH}$ that was observed by the Advanced LIGO, e.g., GW150914 (Abbott et al. 2016a) and GW170104 (Abbott et al. 2017a). Initially the SMA of the BBH is fixed to $a=0.1 \mathrm{AU}$ or $a=$ $0.2 \mathrm{AU}$. The initial eccentricity follows a thermal distribution $f\left(e_{1}\right) \propto e_{1}$, or we simply set $e_{1}=0$. To reduce the effect of GW orbital decay by the binary itself, we set the maximum of $e_{1}$ such that the binaries have a long GW decay time, i.e., with $T_{\mathrm{GW}}>1 \mathrm{Gyr}$.

We assume that the initial eccentricity of the outer orbit of the BBHs follows $f\left(e_{2}\right)=2 e_{2}$, and a random initial orientation of both the inner and the outer orbits of the BBHs. For each model, we perform a series of simulations according to the method described in Section 2, with all or some of the dynamical effects being considered. We can turn on the dynamical effects that we are interested in, and then off, and see the effect of the difference on the results. The results are shown in the following sections.

\subsection{The dynamical evolutions of the BBHs}



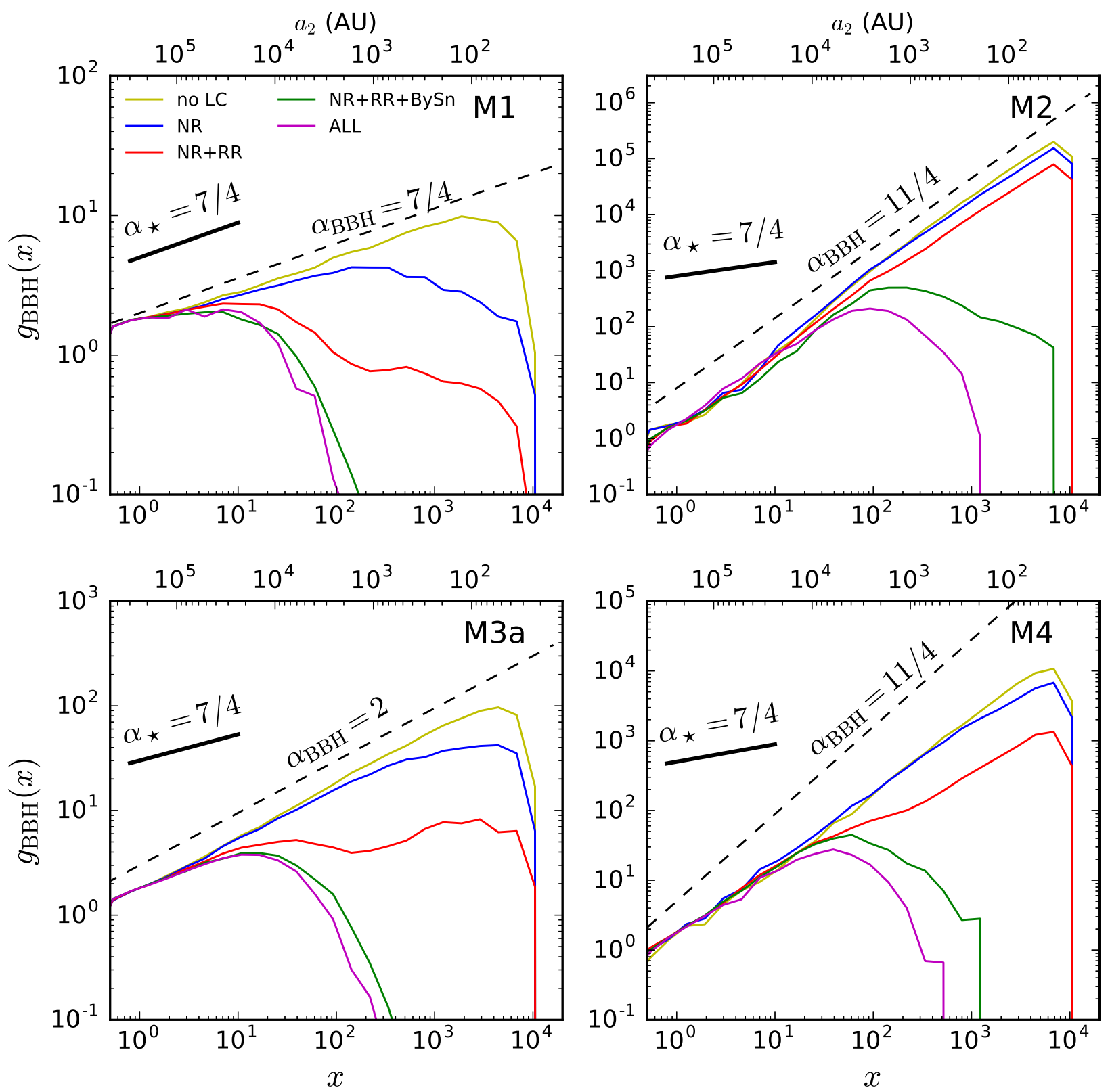

Figure 4. The dimensionless distribution function of the energy of the BBHs' outer orbit, i.e., $g_{\mathrm{BBH}}(x)$ (see Equation 18) in four of the models in Table 2. $x=E / E_{0}$ is the dimensionless energy. The lines in different colors and linestyles show results when different dynamical processes are being considered: The yellow solid line is the result considering only the non-resonant (NR) relaxations and without loss cone; The blue, red, green and magenta solid lines are the result considering the loss cone effect. The text in the legend show the effects being considered: "NR" means non-resonant relaxation, "RR" means resonant relaxation, "BySn" means the binary-single encounter, "ALL" means all of the above effects, and additionally the KL effect, the GW orbital decay of the inner orbit of BBHs. The black dashed line shows the theoretical expectation for a no lose cone case, given by Equation 23, with the powerlaw index given in each panel. The black thick solid line shows the density profile of the background stars $\left(\alpha_{\star}=7 / 4\right)$. 

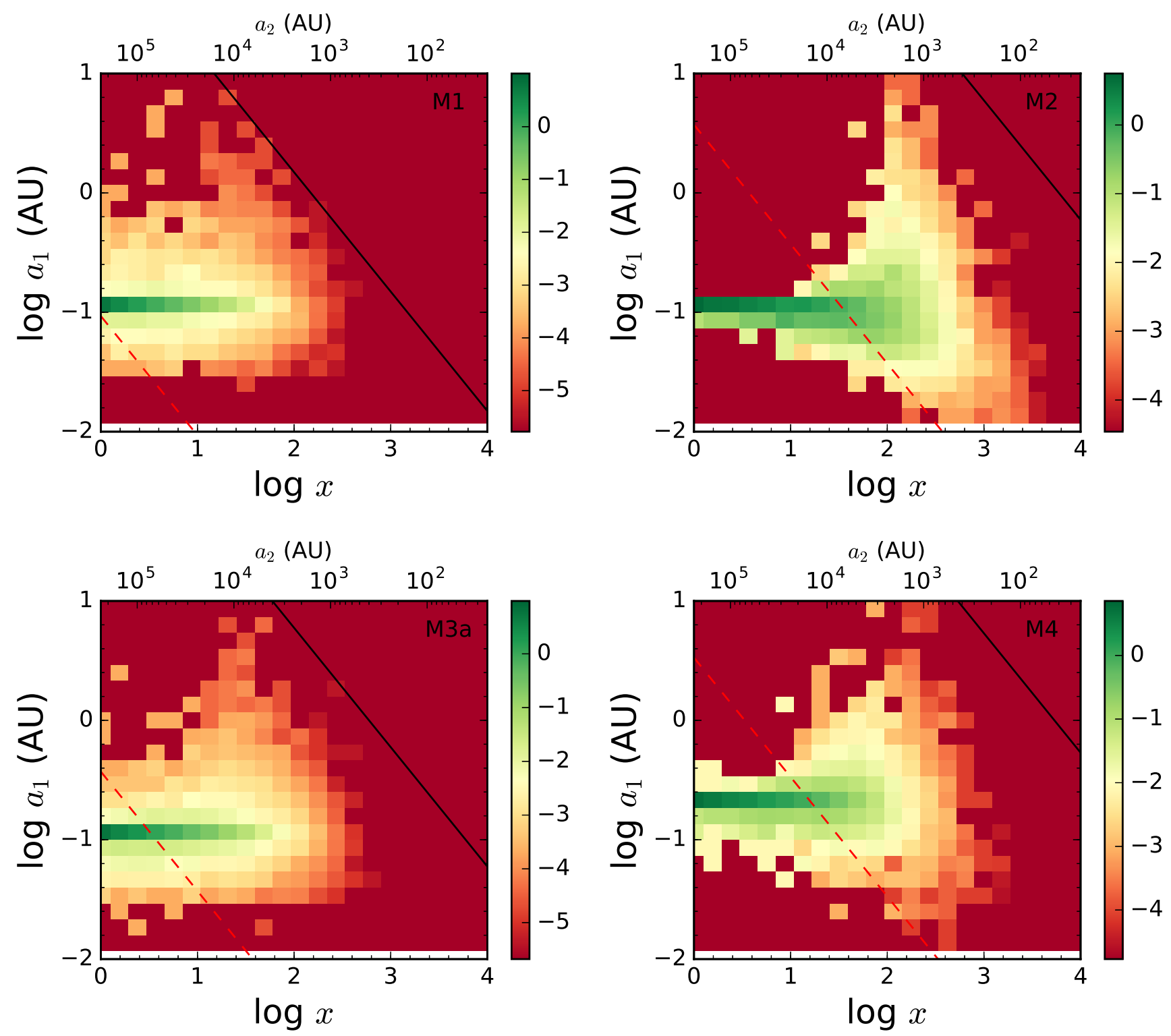

Figure 5. The distribution of the BBHs in the $\log a_{1}-\log x$ space for models M1, M2, M3a and M4. Here $x=E / E_{0}$ is the dimensionless energy. The color contours show the number density of BBHs in log scale per dex ${ }^{-2}$. Note that the non-resonant, resonant relaxations and the binary-single encounters are considered, but other effects (KL and GW orbital decay) are ignored. The red dashed line shows the position where $\xi=1$, which separates the soft (above the line) and hard (below the line) binary regions. The black solid line corresponds to $\epsilon=10^{-3} m_{\star}|E|$. In regions above this line, the binary is considered to be too soft to exist. 

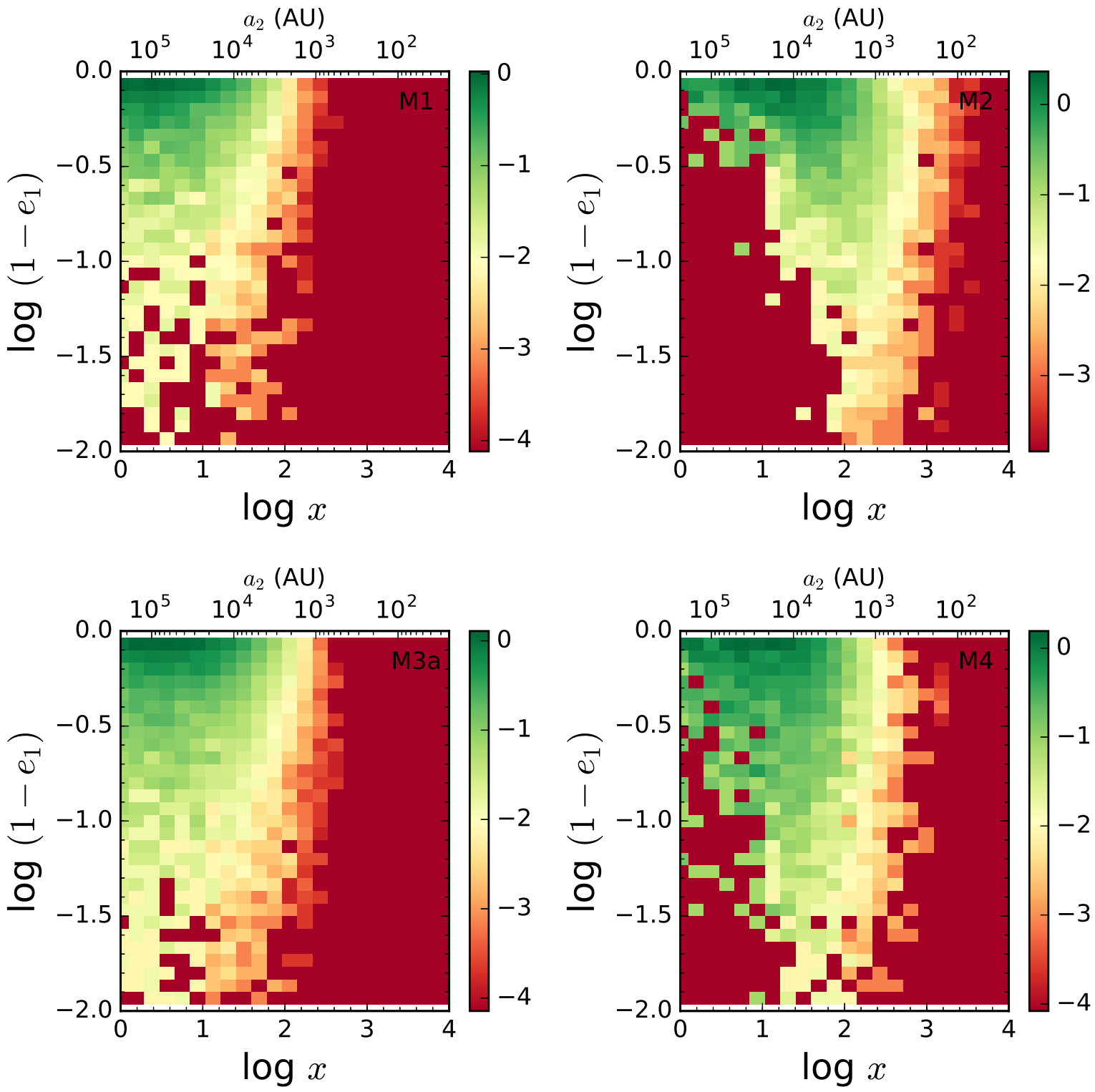

Figure 6. Similar to Figure 5 but for $\log \left(1-e_{1}\right)-\log x$. Here $e_{1}$ is the eccentricity of the inner orbit of the BBHs.

As the number density is dominated by the field stars, the expected density profile of the BBHs should be the same as that of the field stars if $m_{\mathrm{BBH}}=m_{\star}$ and show mass segregation effects if $m_{\mathrm{BBH}}>m_{\star}$. The expected index of the density profile is given by (Alexander \& Hopman 2009)

$$
\alpha_{\mathrm{BBH}}=\left\{\begin{array}{cl}
3 / 2+m_{\mathrm{BBH}} /\left(4 m_{\star}\right) & \text { if } m_{\mathrm{BBH}} / m_{\star} \lesssim 4 \\
9 / 2-\alpha_{\star} & \text { if } m_{\mathrm{BBH}} / m_{\star} \gtrsim 4
\end{array}\right.
$$

The yellow solid lines in Figure 4 show the distribution function $g_{\mathrm{BBH}}(x)$ in model M1, M2, M3a, and M4 when only the NR is considered, and without the loss cone. For model M1, in which the mass of the binary
$m_{\mathrm{BBH}}=10 M_{\odot}$ is equal to the field stars, the density profile of BBHs is the same as that of the field stars, i.e., $\alpha_{\star}=7 / 4$, consistent with Euqation 23. For models M2, M3a, and M4, the density profile of the BBHs also follows Equation 23, consistent with the theoretical expectations.

When additionally the loss cone is considered, the results of density profile in different models are shown with the blue solid lines in Figure 4. We can see that the loss cone can reduce the number of BBHs in the inner regions. The RR process can cause additional decrease of BBHs in the inner regions (see the red solid lines in Figure 4), as also suggested in other studies (e.g., Hopman \& Alexander 2006). This is because the RR 
can excite the eccentricity of the outer orbit of BBHs and move them more efficiently to the loss cone regions. The decrease of BBHs due to the NR or RR effects is only significant when $m_{\mathrm{BBH}} \simeq m_{\star}$. If $m_{\mathrm{BBH}} \gtrsim 4 m_{\star}$, the $\mathrm{BBH}$ destroyed in loss cone are quickly replenished by the $\mathrm{BBHs}$ moving from other regions under the strong mass segregation effect.

The binary-single encounters can reduce further the number of BBHs in the inner region (see the green lines in Figure 4), mainly because the BBHs in the inner regions become soft binaries in all models. We define a dimensionless parameter $\xi$ (Hopman 2009),

$$
\xi=\frac{\epsilon}{m_{\star} \sigma^{2}} \simeq \frac{m_{A} m_{B} a_{2}}{2 m_{\star} M_{\bullet} a_{1}} .
$$

Then the binary is soft if $\xi \ll 1$ and hard if $\xi \gg 1$. Table 2 shows the value of $\xi$ at $a_{2}=r_{h}$. We can see that for models M1 and M3a-c, within the cluster the BBHs are mostly soft binaries. For models M2 and M4, the binary is hard near the edge of the cluster. However, they will be soft in the inner regions of the cluster.

The soft binaries are likely to be ionized after encounter. Figure 5 shows the distribution of the SMA of the inner orbit of the BBHs for different models (note that KL and GW orbital decay are still ignored in these figures). We can see that in the hard binary region, i.e., $\xi>1$, the binary always becomes harder after encounter. However, in the soft binary region, the binarysingle encounters can increase the SMA $\left(a_{1}\right)$ of the inner binary. For model M2, as $m_{\mathrm{BBH}} \gg m_{\star}$, a significant number of BBHs sink into the inner regions of the cluster under strong mass segregation effect, where the rates of binary-single encounters are dramatically increased. In all models, in the soft binary region, such encounters can still decrease $a_{1}$ down to $10^{-2} \mathrm{AU}$, which will help increase the event rates of GW mergers of the BBHs.

Similarly, Figure 6 shows the distribution of the eccentricity of the inner orbit of the BBHs. We can see that in all models, the eccentricity of the binaries will be dramatically changed due to the binary-single encounters. The maximum eccentricity can be up to $\gtrsim 0.99$, which will further increase the event rate of GW mergers of the BBHs.

The magenta lines in Figure 4 show the density distribution of BBHs when all of the dynamical effects (including the vector RR relaxation, KL oscillations and the orbital decays due to GW radiations) are considered. Comparing the green lines with the magenta lines in Figure 4, we find that considering the GW orbital decay and KL effects can reduce further the number of BBHs in the inner regions, which means that many BBHs are merged. We find that the vector $\mathrm{RR}$ relaxation process has insignificant effects on the number density of BBHs. In all models, the BBHs can survive if they are
$10^{2}-10^{4} \mathrm{AU}$ away from the MBH Only in the model M2, where the mass segregation effects are the most significant, the BBHs can sink into more inner region with the distance of $\sim 100 \mathrm{AU}$ from the $\mathrm{MBH}$.

We find that the density profile of BBHs in models with $\alpha_{\star}=1$ (e.g., M3b) is similar to those of $\alpha_{\star}=7 / 4$, and only in the case that $m_{\mathrm{BBH}} \gg m_{\star}$, we find that the $\mathrm{BBH}$ are more concentrated in the central regions for the case $\alpha_{\star}=1$. These simulation results are consistent with what is expected in Equation 23.

\section{2. $G W$ mergers of $B B H s$}

There are a number of possible dynamical channels to merge BBHs in the cluster containing a $\mathrm{MBH}$. Here we exemplify three of them, which are presented in Figures 7-9. The details are described as follows.

In the first case, the BBHs experience hundreds to thousands times of binary-single encounters, and then merge under the combined effect of KL oscillations and the binary-single encounters. This channel is more common for those models with strong mass segregation effects, i.e., $m_{\mathrm{BBH}} \gg m_{\star}$. Figure 7 shows an example of the evolutions of the BBH's inner and outer orbits in model M2. The outer orbit of the BBH first gradually migrates from the outer parts of the cluster $\left(a_{2} \sim\right.$ $\left.10^{5} \mathrm{AU}\right)$ into the inner regions $\left(a_{2} \sim 10^{3} \mathrm{AU}\right)$. Along the way the binary-single encounters become more and more frequent. Each binary-single encounter changes the inner orbit slightly, as the incoming field star is much lighter than the components of the BBHs, i.e., $m_{A}, m_{B} \gg m_{\star}$. From the top right panel of Figure 7 , we can see that the encounters gradually increase the $e_{1}$ of the $\mathrm{BBH}$ up to $\sim 0.8-0.9$, and the SMA of the $\mathrm{BBH}$ also increases up to about $2 \mathrm{AU}$. When the pericenter of the BBH's outer orbit approaches to about tens of $r_{t}$, the KL effects become important, which can increase $e_{1}$ rapidly. Note that simultaneously the binary-single encounters also change the inner orbits. Under these two effects, the inner orbit of the $\mathrm{BBH}$ finally reaches to a very high eccentricity and eventually the $\mathrm{BBH}$ merges due to GW radiations.

The bottom panels of Figure 7 show the evolutions of the orbital orientations of both the inner and outer orbits of the $\mathrm{BBH}$. The orientation of the outer orbit evolves under vector RR and the KL effect (bottom left panel of Figure 7). The orientation of the inner orbit is changed cumulatively during multiple binary-single encounters, and becomes quite rapidly during the $\mathrm{KL}$ precesses (bottom right panel of Figure 7). The rapid oscillations of the inner orbital orientations can help to trigger the merging of BBHs by KL effect. For example, before merging, $I_{2} \sim \pi$ and $I_{1} \sim \pi / 2$, the relative inclination between the inner and outer orbits is close to $\pi / 2$, which can help to enhance the eccentricity oscillations 

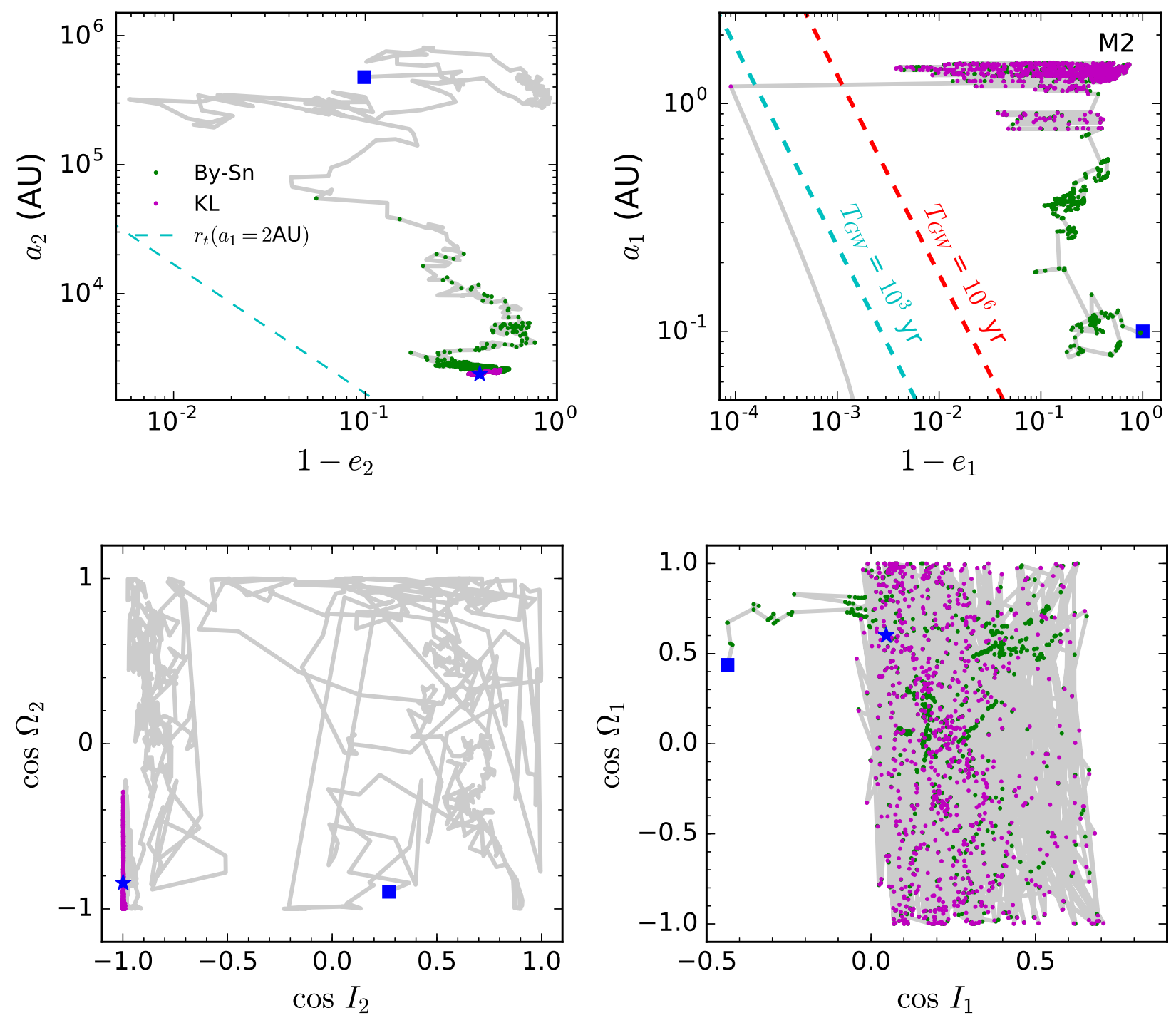

Figure 7. An example of evolutions of BBH's inner and outer orbits. Left panels: The evolutions of the outer orbit of the $\mathrm{BBH}$. The dashed cyran line in the top left panel shows the tidal radius for the BBH with $a_{2}=2 \mathrm{AU}$. In the bottom left panel, the evolution of $\Omega_{2}$ and $I_{2}$ are mainly driven by vector RR. Right panels: The evolutions of the inner orbit of the BBH. The dashe lines in the top right panel show the GW timescales. "By-Sn" means the position after the binary-single encounter; "KL" means the position after the KL oscillations. The filled blue square symbol marks the starting position of the BBHs in each panel. The filled blue star symbol in all panels marks the position where the BBH is merged. The initial condition of the model is given in model M2.

and merging the BBHs (Wen 2003).

Figure 8 shows another example of the BBH's evolutions in model M2. In Figure 8, the SMA of the BBH always tends to decrease due to multiple binary-single encounters. This is because the BBH is mostly remaining in the hard binary regions, and the binary-single encounters always decrease the SMA for a hard binary. A hard binary makes the frequency of binary-single encounters drops and prevents another encounter even if it reaches the inner regions.

Different from model M2, M3a assumes more massive background field stars. Such initial conditions have two consequences: (1) The change of the inner orbit of the BBHs can be dramatic. (2) The number of encounters is much smaller in M3a than in M2, as the number of field stars is much smaller (due to their massiveness) according to Equations 14 or 15. In these cases, the BBHs can experience only a few binary-single encounters, which will dramatically increase the eccentricity of the binary, and soon lead to merger due to the GW radiations.

In some rare cases, the GW merging event can be triggered due to the multiple encounters between the $\mathrm{BBH}$ and the $\mathrm{MBH}$, as shown in Figure 9. In the final revolution, from the bottom panels of Figure 9 we find 

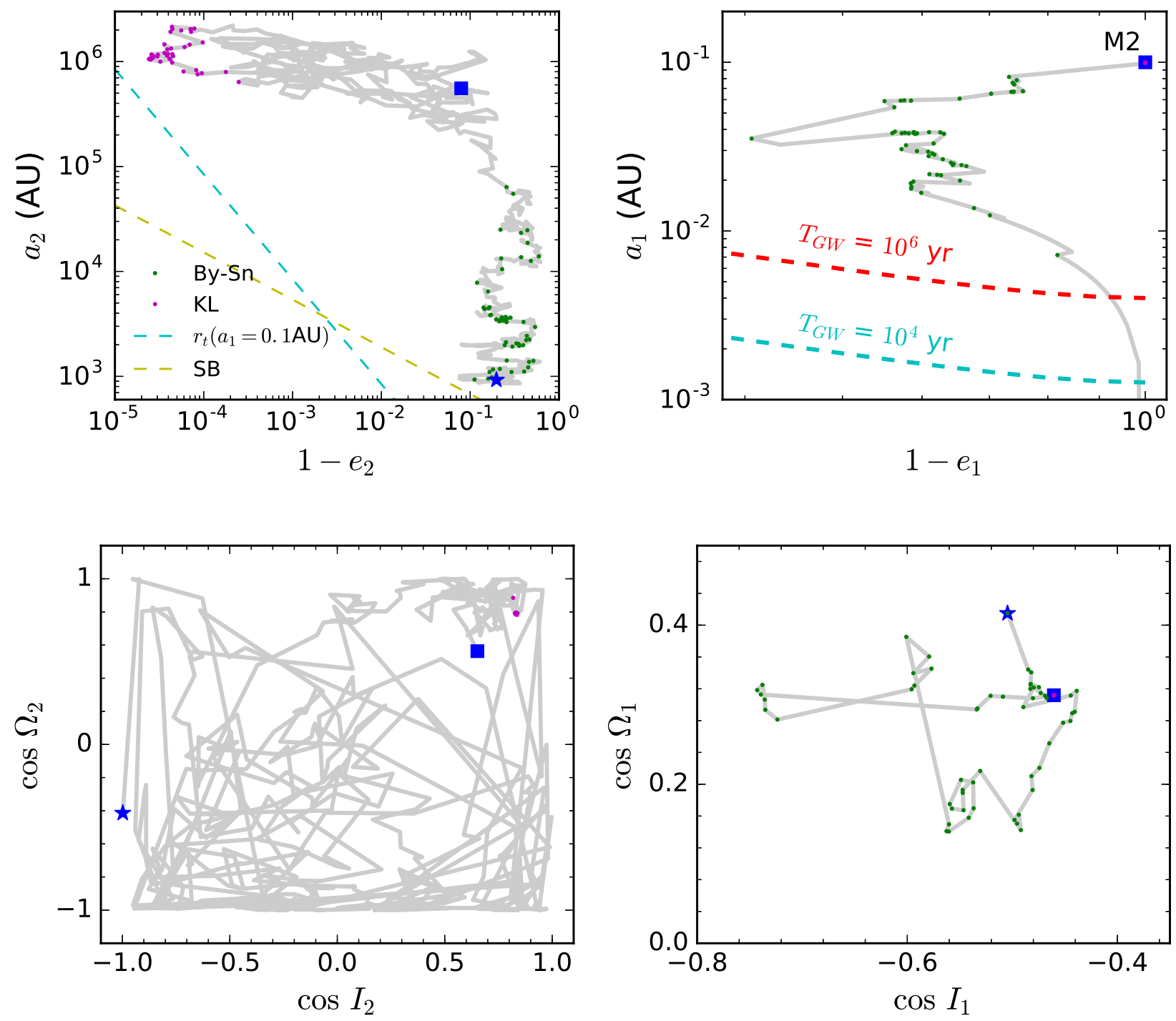

Figure 8. Similar to Figure 7 but for a different BBH, where the initial condition is given by model M2. The dashed yellow line shows the Schwarzschild barrier, below which the RR is suppressed.

that the relative inclination between the plane of the inner and outer orbits is $\sim \pi / 2$. As the tidal force of $\mathrm{MBH}$ is nearly perpendicular to the orbital plane of the BBH, it tends to suppress the BBH (Zhang et al. 2010) and help to merge the BBH.

This usually happens when the BBH is at the outer parts of the cluster, such that it can approach to the loss cone region without being ionized by the binarysingle encounters, and that the effect of KL oscillations is not so significant. However, we notice that if the binary-single encounters are switched off, then this case is very frequent. The eccentricities of the $\mathrm{BBHs}$ can be excited to very high values due to the multiple encounters between $\mathrm{BBH}$ and $\mathrm{MBH}$, and the $\mathrm{GW}$ is so strong that they can merge before the next encounters with the $\mathrm{MBH}$.
In all models, we find that the SB does not affect much the steady-state flux of binaries into the loss cone, which may be important for those single stars (Bar-Or \& Alexander 2016). As we can see from Figures 8 and 9 , in most cases $\mathrm{SB}$ is below the tidal radius of $\mathrm{BBHs}$ with $a_{1} \gtrsim 0.1 \mathrm{AU}$, and the $\mathrm{SB}$ is only effective if $a_{2} \lesssim 300 \mathrm{AU}$, where most BBH can not penetrate into. As RR is effective below $a_{2} \sim 10^{4} \mathrm{AU}$ (See Figure 1), the tidal rates of BBHs are not affected much by the $\mathrm{SB}$, and the $\mathrm{RR}$ process can still drive most of the BBHs effectively into loss cone regions.

At the end of the simulation, suppose that the number of integrated binaries that survived in the simulation is $N$, and the rate of merging events is $\dot{N}_{e}=d N_{e} / d t$, where $d N_{e}$ is the total number of merging events during time span $d t$. We can define a normalized merging event rate 

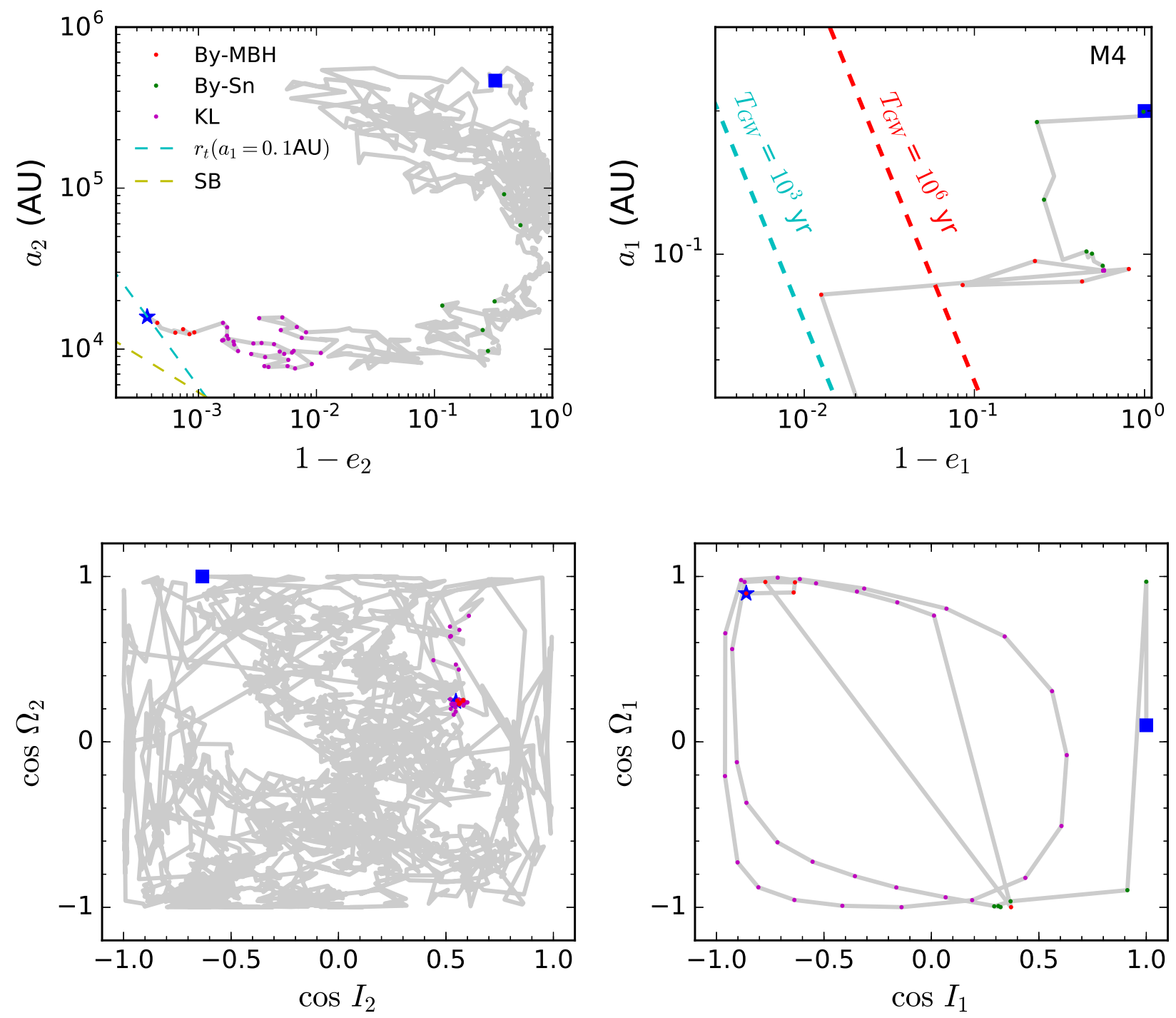

Figure 9. Similar to Figure 7 but for a different BBH, where the initial condition is given by model M4. "By-MBH" means the position after the binary-MBH encounter. The dashed yellow line shows the Schwarzschild barrier, below which the RR is suppressed.

in the nucleus cluster, $\mathscr{R}=\dot{N}_{e} / N$. It means that if we observed only one $\mathrm{BBH}$ in the real galactic nucleus clusters at current moment, the merging event rate in such cluster is then given by $\mathscr{R}$. Note here that $N$ and $\dot{N}_{e}$ are calculated after considering the weight of each event in the clone scheme (see Section 2.5).

Based on $\mathscr{R}$, the merging rate of the BBHs in a real galactic nucleus can then be estimated after some scaling with the realistic number of BBHs in each galactic nucleus. If assuming a constant number fraction, i.e., $f_{n b}$, of BBHs in the cluster with respect to the field stars, the event rate is given by

$$
R=\frac{f_{n b} M_{\bullet}}{m_{\star}} \mathscr{R} .
$$

If alternatively assuming a constant mass fraction, i.e., $f_{m b}$, of BBHs with respect to the field stars, it becomes

$$
R=\frac{f_{m b} M_{\bullet}}{\left\langle m_{\mathrm{BBH}}\right\rangle} \mathscr{R},
$$

where $\left\langle m_{\mathrm{BBH}}\right\rangle$ is the mean mass of the BBHs.

In this work, we assume that the mass and the number fraction of all stellar black holes are much smaller than the field stars. If assuming a constant mass fraction, we set $f_{m b}=10^{-3}$. If assuming a constant number fraction of BBHs, we set $f_{n b}=\min \left(10^{-2} m_{\star} /\left\langle m_{\mathrm{BBH}}\right\rangle, 10^{-3}\right)$, such that the total mass of the BBHs does not exceed $\sim 0.01 M_{\bullet}$, which is likely the mass fraction of stellarmass black holes in a nucleus cluster under standard initial mass function of stars (Hopman \& Alexander 2006).

Table 3 shows the estimated GW event rates according to Equation 25 and Equation 26 in different 
Table 3. Dependence of the merging rate of BBHs on different dynamical effects

\begin{tabular}{lcccc}
\hline model & ALL & Vec RR off & KL off & By-Sn off \\
\hline M1 & $3.3 \pm 0.2^{a}$ & $2.5 \pm 0.2$ & $2.6 \pm 0.2$ & $1.5 \pm 0.2$ \\
& $3.3 \pm 0.2^{b}$ & $2.5 \pm 0.2$ & $2.6 \pm 0.2$ & $1.5 \pm 0.2$ \\
M2 & $1046 \pm 76$ & $939 \pm 72$ & $899 \pm 70$ & $362 \pm 48$ \\
& $104.6 \pm 7.6$ & $93.9 \pm 7.2$ & $89.9 \pm 7.0$ & $36.2 \pm 4.8$ \\
M3a & $11.1 \pm 0.3$ & $11.0 \pm 0.3$ & $10.5 \pm 0.3$ & $3.3 \pm 0.2$ \\
& $5.5 \pm 0.1$ & $5.5 \pm 0.1$ & $5.3 \pm 0.1$ & $1.7 \pm 0.1$ \\
M3b & $9.0 \pm 0.4$ & $7.8 \pm 0.4$ & $8.2 \pm 0.4$ & $3.3 \pm 0.2$ \\
& $4.5 \pm 0.2$ & $3.9 \pm 0.2$ & $4.1 \pm 0.2$ & $1.7 \pm 0.1$ \\
M3c & $22.1 \pm 0.9$ & $19.6 \pm 0.8$ & $20.3 \pm 0.8$ & $6.9 \pm 0.5$ \\
& $11.0 \pm 0.4$ & $9.8 \pm 0.4$ & $10.1 \pm 0.4$ & $3.4 \pm 0.2$ \\
M4 & $169 \pm 10.6$ & $175 \pm 11$ & $163 \pm 10$ & $48 \pm 5.7$ \\
& $28.1 \pm 1.8$ & $29.3 \pm 1.8$ & $27.3 \pm 1.7$ & $8.0 \pm 1.0$ \\
\hline
\end{tabular}

Note - Note that the rates in this table are only for the purpose of demonstrating the effects of different dynamical processes. For each model, we have assumed Milky-Way-like galaxies with MBH $M_{\bullet}=4 \times 10^{6} M_{\odot}$, and have oversimplified initial conditions of the BBHs (see

Table 2). For more realistic event rate estimations in the local universe, see Section 4 or Table 5.

${ }^{a}$ Merging rates in unit of $\mathrm{Gyr}^{-1}$, assuming a constant number fraction of $\mathrm{BBHs}$, i.e., $f_{n b}=\min \left(10^{-3}, 10^{-2} m_{\star} /\left\langle m_{\mathrm{BBH}}\right\rangle\right)$

${ }^{b}$ Merging rates in unit of $\mathrm{Gyr}^{-1}$, assuming a constant mass fraction of BBHs, i.e., $f_{m b}=10^{-3}$

models. The first row for each model shows the result if assuming a constant number fraction of BBHs and $f_{n b}=\min \left(10^{-3}, 10^{-2} m_{\star} /\left\langle m_{\mathrm{BBH}}\right\rangle\right)$ while the second row assuming a constant mass fraction of BBHs and $f_{m b}=10^{-3}$. We can see that, if all the dynamical effects are considered, the event rates range from $1-10^{3} \mathrm{Gyr}^{-1}$ in the six models investigated here. Merging rates assuming a constant number fraction are usually larger than those assuming a constant mass fraction, as usually the field stars are considered lighter than the BBHs. The change of $\alpha_{\star}$ parameter does not affect significantly the merging rates, as it changes only the density profiles in the cases that $m_{\mathrm{BBH}} \gg m_{\star}$. If alternatively the initial eccentricity is not zero, but follows the thermal distribution, the merging rates will be about twice larger. This can be straight forwardly obtained as the larger the eccentricity, the easier the BBHs can be merged.

We find that the exchange of the $\mathrm{BBH}$ with the incoming stars is common in some models, e.g., M1, M3a, as usually the exchange event is frequent if $m_{\star} \sim m_{\mathrm{BBH}}$. These exchange events decrease the total GW event rate significantly. By removing the exchanged BBHs from the simulation, we find that the GW event rate is about twice smaller.

As we have shown in Figures 7-9, the mergers of BBHs are results of the combinations of various dynamical effects. To explore their importance in the merger of BBHs, we also run similar simulations for each model, but excluding some dynamical processes. The results are shown in Table 3. We do not see a clear difference when the vector RR effect is being considered or not. If the vector $\mathrm{RR}$ effects are switched off, the event rates usually drop only slightly for all models. As we consider only $M_{\bullet}=4 \times 10^{6} M_{\odot}$ in this section, this is consistent with Hamers et al. (2018), that the vector RR effect is more important for MBHs with $M_{\bullet}=10^{4}-10^{5} M_{\odot}$, but quickly becomes negligible for massive $\mathrm{MBHs}$.

Many of the BBHs are ionized or tidally disrupted along the way from the outer to the inner regions. Thus, the KL effect does not contribute much to the total merging rate as few of the $\mathrm{BBHs}$ can survive to the vicinity of $\mathrm{MBH}$ where it is most efficient. If the $\mathrm{KL}$ effects are switched off, we do not see a significant difference on the GW merging rates (See Table 3). On the other hand, many of the BBHs may coalesce due to multiple binary-single encounters. Our results suggest that the binary-single encounters can be an important channel that leads to the mergers of the BBHs.

If we switch off the binary-single effects, then the inner orbits of BBHs are affected only by KL oscillations or the strong encounters with the MBH. We find that in these cases, most of the BBHs will merge after the excitement of eccentricity due to both the KL oscillations and the BBH-MBH encounters. Note that the contribution from the latter one to the merging rates is only significant when the binary-single encounters are switched off. However, as these two effects are less efficient in merging the BBHs than those of the binary-single encounters, there is a significant drop in the merging rates for all models (see results in Table 3).

We find that the BBHs in our simulations are all affected by the dynamical effects mentioned above before they merged. We do not find any $\mathrm{BBH}$ end up with merger by evolving isolatedly ${ }^{3}$ for all models except M3c. In model M3c, the merging rate of BBHs evolved isolatedly is $\sim 0.5 \mathrm{Gyr}^{-1}$. Thus, the merging rates in Table 3 are unique results for BBHs in Galactic nuclei and are not affected by BBHs evolved in isolation.

\subsection{The eccentricity of the BBH mergers in the LIGO band}

The BBH remains some eccentricity when the frequency of its gravitational wave enters into the LIGO

\footnotetext{
3 Hereafter "merger by evolving isolatedly" means that the merging of $\mathrm{BBH}$ is caused only by the $\mathrm{GW}$ orbital radiation in the simulation, and that the $\mathrm{BBH}$ does not experience any other dynamical process, including the binary-single encounters, binary$\mathrm{MBH}$ encounters or KL oscillations, etc, before the merging event happens.
} 

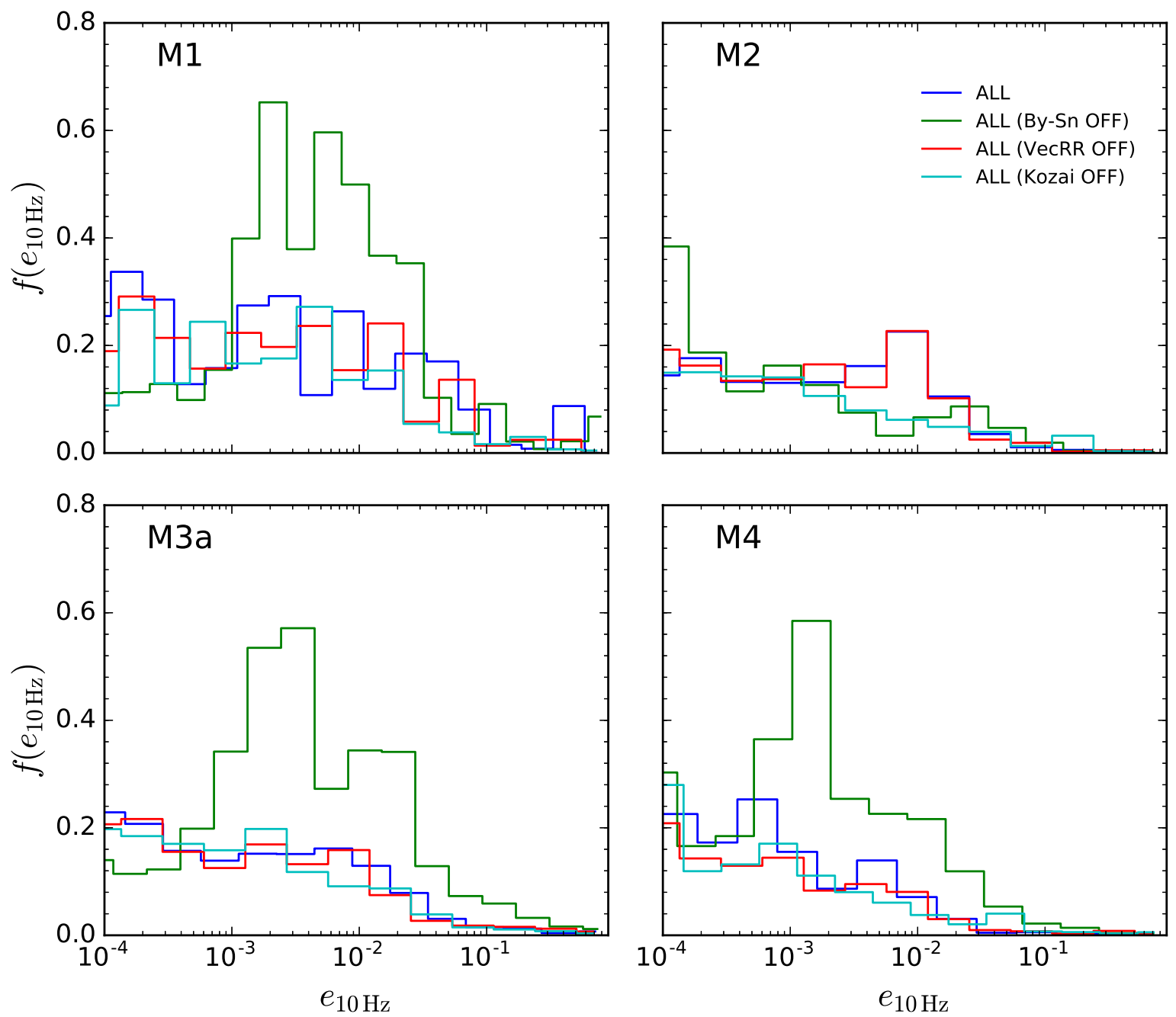

Figure 10. The distribution of eccentricity of BBHs when its peak gravitational wave frequency enters into the LIGO band, i.e., $f_{\mathrm{GW}}=10 \mathrm{~Hz}$. The solid lines show the PDF. The blue lines are the results when all the dynamical effects are included, while the green, red, and cyan lines are the results when binary-single encounter, vector RR or KL effects only, is turned off, respectively.

band, i.e., $f_{\mathrm{GW}}=10 \mathrm{~Hz}$. We denote the eccentricity at this moment as $e_{10 \mathrm{~Hz}}$. For BBHs in the galactic field, their orbital eccentricities are very close to zero when entering the LIGO band (Peters 1964). If a BBH has a significant residual eccentricity, say $e_{10 \mathrm{~Hz}} \gtrsim 0.1$, the modulation in the waveform will be easily recognized by the matched-filtering techniques (Hinderer \& Babak 2017). In contrast, as we will see below, the BBHs from galatic nuclei have a non-negligible possibility to have a significant $e_{10 \mathrm{~Hz}}$. This will be a smoking-gun effect to distinguish $\mathrm{BBH}$ mergers from galactic fields and nucleus clusters when enough events are detected to perform a statistical study.

As we mentioned in earlier sections, the excitement of the eccentricity of BBHs is mainly through binary-single encounters, BBH-MBH multiple encounters, or KL oscillations. Different channels excite the eccentricity of
Table 4. Fraction of eccentric BBHs in nucleus clusters when $f_{\mathrm{GW}}=10 \mathrm{~Hz}$

\begin{tabular}{lcccc}
\hline model & ALL & Vec RR off & KL off & By-Sn off \\
\hline M1 & $10.3^{a}\left(1.7^{b}\right)$ & $12.1(2.5)$ & $7.3(1.8)$ & $21.6(4.9)$ \\
M2 & $4.2(0.46)$ & $4.3(0.79)$ & $4.1(1.5)$ & $5.5(0.76)$ \\
M3a & $5.5(1.1)$ & $4.4(1.4)$ & $4.6(1.0)$ & $18.5(4.1)$ \\
M3b & $2.8(1.2)$ & $2.5(0.5)$ & $1.1(0.4)$ & $8.8(3.3)$ \\
M3c & $3.7(0.7)$ & $2.6(0.4)$ & $3.8(0.7)$ & $14(4.4)$ \\
M4 & $1.4(0.3)$ & $1.7(0.6)$ & $2.8(0.6)$ & $7.7(1.2)$ \\
\hline
\end{tabular}

Note- ${ }^{a}$ Percentage of events with $e_{10 \mathrm{~Hz}}>0.01$

${ }^{b}$ Percentage of events with $e_{10 \mathrm{~Hz}}>0.05$.

the BBHs in different ways and to different degrees, and thus the distribution of $e_{10 \mathrm{~Hz}}$ depends on each or combinations of the above three effects: (1) Binary-single encounters excite $e_{1}$ dramatically if $m_{\star} \sim m_{\mathrm{BBH}}$, and 
slowly if $m_{\star} \ll m_{\mathrm{BBH}}$. When this effect is switched on, the excitement of $e_{1}$ due to BBH-MBH encounters mentioned below will be significantly suppressed. (2) BBH-MBH encounters excite $e_{1}$ dramatically if the encounter is close, e.g., $r_{p} \lesssim r_{t}$, and smoothly if $r_{p} \gtrsim$ $r_{t}$ (Zhang et al. 2010). Models with massive field stars have large perturbations in the outer orbits of BBHs and the BBHs can move fast in the lose cone region. Thus, for these models their excitement of $e_{1}$ can be dramatic. (3) The KL effect can excite $e_{1}$ to very high values, however, only if some preferred orbital configurations are satisfied (especially for the inclination angle). In most cases, the KL effect changes $e_{1}$ very smoothly.

Figure 10 shows their probability distribution function (PDF) of $e_{10} \mathrm{~Hz}$ when all dynamical effects are considered or without some of the specific ones. Table 4 shows the fraction of BBHs merged with $e_{10 \mathrm{~Hz}}>0.01$ (or $e_{10 \mathrm{~Hz}}>0.05$ ) in different models. When all the dynamical effects are included, we find that there are $\sim 2-15 \%(0.3-5 \%)$ of the BBHs have $e_{10 \mathrm{~Hz}}>0.01$ $\left(e_{10 \mathrm{~Hz}}>0.05\right)$. M1 has the highest probability as in this model the binary-single encounters excite $e_{1}$ very dramatically. Note that KL effects can affect the $e_{1}$ of $\mathrm{BBHs}$, however, it is not the most possible final merging channel in all models. Thus, switching it off does not affect much of the PDF of $e_{10 \mathrm{~Hz}}$. We also do not find significant difference by switching vector $R R$ off in the simulation.

If the effect of binary-single encounter is switched off, we can see that for all models (except M2), the $e_{10 \mathrm{~Hz}}$ is higher than the case when the binary-single encounter is switched on. For these models (except M2), the BBH$\mathrm{MBH}$ multiple encounters can excite the eccentricity of BBHs very dramatically, such that $e_{1}$ can be very high. For model M2, as $m_{\star} \ll m_{\mathrm{BBH}}$, the outer orbits of BBHs are perturbed very smoothly, such that they move in and out of the KL region very frequently $\left(3 r_{t}<r_{p}<\right.$ $\left.\min \left[20,0.58\left(T_{\mathrm{GR}} / P 2\right)^{2 / 3}\right] r_{t}\right)$. In some rare cases the $\mathrm{KL}$ excites the $e_{1}$ of BBHs to very high values and thus they merge quickly. However, in most cases the BBHs move out of the KL region and their eccentricity are changed only to median values. They then evolve isolatedly and merge before it enters the KL region again, thus the overall $e_{10 \mathrm{~Hz}}$ remains low.

\section{THE MERGING EVENTS OF BBHS IN THE LOCAL UNIVERSE}

The event rates shown in Table 3 are estimated only for Milky-Way-like galaxies, and are unrealistic due to their over simplification of the initial conditions of the $\mathrm{BBH}$ populations. In this section, we calculate a more realistic estimation of the event rates basing on population synthesis.

The observed GW rate of merging BBHs is about
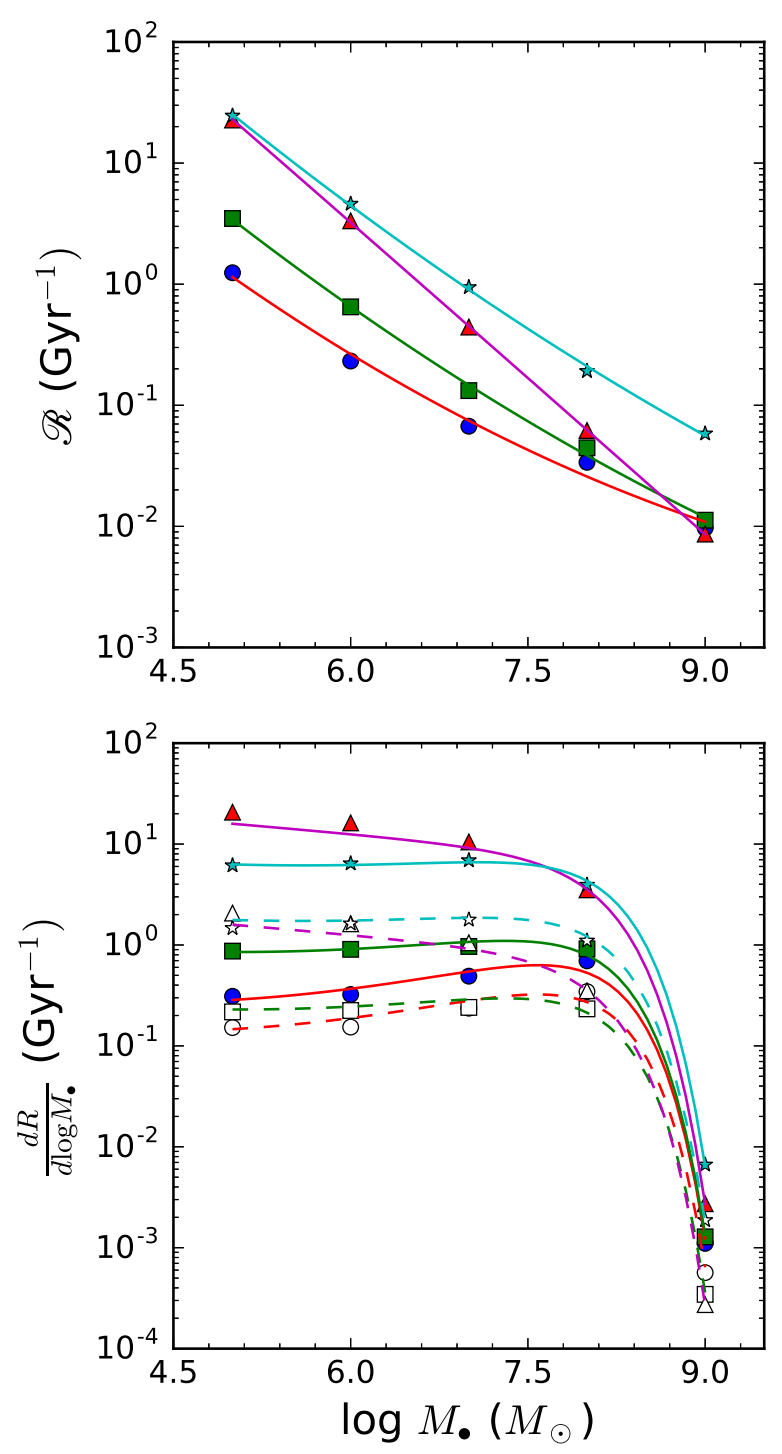

Figure 11. Top panel: The normalized event rate $\mathscr{R}$ for galaxies containing a $\mathrm{MBH}$ with mass $M_{\bullet}$. Bottom panel: The differential merging rate as a function of the MBH mass. We assume $\alpha_{\star}=7 / 4$. The blue, green, magenta and cyan symbols are the numerical results for models MPW10 series, MUN10 series, MUN1 series and MUR10 series, respectively. The color lines are the fitting results. In the bottom panel, the solid lines are for models assuming $f_{n b}=$ $\min \left(10^{-3}, 10^{-2} m_{\star} /\left\langle m_{\mathrm{BBH}}\right\rangle\right)$ while the dashed lines are for models assuming $f_{m b}=10^{-3}$.

12-213 $\mathrm{Gpc}^{-3} \mathrm{yr}^{-1}$ (Abbott et al. 2016d, 2017a). To compare our simulation results with the observations, we assume two possible cases of the mass distribution in Abbott et al. (2016d): (1) a logarithmicuniform distribution (hereafter the "UN" model), i.e., $f\left(m_{A}, m_{B}\right) \propto m_{A}^{-1} m_{B}^{-1} ;(2)$ a power-law distribution of primary mass and a uniform distribution on the secondary mass (hereafter the "PW" model), i.e., $f\left(m_{A}\right) \propto$ $m_{A}^{-2.35}, f\left(m_{B}\right) \propto m_{B}^{-1}$. In both distributions we require $5 M_{\odot}<m_{B} \leq m_{A}$ and $m_{A}+m_{B}<100 M_{\odot}$. 
Table 5. Models

\begin{tabular}{|c|c|c|c|c|c|c|c|c|c|c|}
\hline Model & $\mathrm{MF}^{a}$ & $r_{i}^{b}$ & $m_{\star}\left(M_{\odot}\right)$ & $\log M \bullet$ & $\left\langle m_{\mathrm{BBH}}\right\rangle$ & $P\left(e_{10 \mathrm{~Hz}}\right)^{c}$ & $\mathscr{R}^{d}$ & $R_{\mathrm{tot}}^{e}$ & $R_{\mathrm{tot}}^{f}$ & $P_{\mathrm{tot}}\left(e_{10 \mathrm{~Hz}}\right)^{g}$ \\
\hline MPW10-5 & PW & $r_{h}$ & 10 & 5 & 21 & $17(1.5)$ & 1.43 & & & \\
\hline MPW10-6 & $\mathrm{PW}$ & $r_{h}$ & 10 & 6 & 21 & $8.6(0.8)$ & 0.24 & & & \\
\hline MPW10-7 & $\mathrm{PW}$ & $r_{h}$ & 10 & 7 & 21 & $2.5(0.9)$ & 0.06 & 1.6 & 0.8 & $4.3(0.7)$ \\
\hline MPW10-8 & $\mathrm{PW}$ & $r_{h}$ & 10 & 8 & 20 & $0.5(0.2)$ & 0.03 & & & \\
\hline MPW10-9 & $\mathrm{PW}$ & $r_{h}$ & 10 & 9 & 19 & $0.1(0.0)$ & 0.01 & & & \\
\hline MUN10-5 & UN & $r_{h}$ & 10 & 5 & 41 & 10.(1.4) & 3.48 & & & \\
\hline MUN10-6 & UN & $r_{h}$ & 10 & 6 & 45 & $5.4(0.7)$ & 0.84 & & & \\
\hline MUN10-7 & UN & $r_{h}$ & 10 & 7 & 43 & $1.9(0.7)$ & 0.12 & 3.2 & 0.9 & $3.5(0.7)$ \\
\hline MUN10-8 & UN & $r_{h}$ & 10 & 8 & 40 & $0.7(0.3)$ & 0.04 & & & \\
\hline MUN10-9 & UN & $r_{h}$ & 10 & 9 & 38 & $0.3(0.1)$ & 0.01 & & & \\
\hline MUR10-5 & UN & $0.1 r_{h}$ & 10 & 5 & 41 & 17.(1.5) & 24.6 & & & \\
\hline MUR10-6 & UN & $0.1 r_{h}$ & 10 & 6 & 39 & 10.(0.9) & 4.61 & & & \\
\hline MUR10-7 & UN & $0.1 r_{h}$ & 10 & 7 & 39 & $5.0(0.5)$ & 0.94 & 20.0 & 5.6 & $7.5(0.8)$ \\
\hline MUR10-8 & UN & $0.1 r_{h}$ & 10 & 8 & 36 & $1.2(0.4)$ & 0.19 & & & \\
\hline MUR10-9 & UN & $0.1 r_{h}$ & 10 & 9 & 36 & $0.3(0.1)$ & 0.06 & & & \\
\hline MUN1-5 & UN & $r_{h}$ & 1 & 5 & 45 & $26(1.6)$ & 21.5 & & & \\
\hline MUN1-6 & UN & $r_{h}$ & 1 & 6 & 35 & $13(1.2)$ & 3.77 & & & \\
\hline MUN1-7 & UN & $r_{h}$ & 1 & 7 & 36 & $6.7(1.6)$ & 0.50 & 30.8 & 3.1 & $12(1.3)$ \\
\hline MUN1-8 & UN & $r_{h}$ & 1 & 8 & 42 & $2.9(0.8)$ & 0.08 & & & \\
\hline MUN1-9 & UN & $r_{h}$ & 1 & 9 & 37 & $1.8(0.6)$ & 0.01 & & & \\
\hline
\end{tabular}

Note - ${ }^{a}$ The assumed mass function of BBHs, where "PW" means power law distribution of primary mass that follows $f\left(m_{A}\right) \propto m_{A}^{-2.35}$, "UN" means logarithmic-uniform distribution of primary mass that follows $f\left(m_{A}\right) \propto m_{A}^{-1}$. The mass functions of the secondary component of these two both follow $f\left(m_{B}\right) \propto m_{B}^{-1}$ (Abbott et al. 2016d).

${ }^{b}$ The initial SMA of the outer orbits of BBHs in the cluster.

${ }^{c}$ Percentage of BBHs with $e_{10 \mathrm{~Hz}}>0.01$ or $e_{10 \mathrm{~Hz}}>0.05$ (in the brackets) in each model.

${ }^{d}$ Normalized merging rate $\left(\mathrm{Gyr}^{-1}\right)$ in a single galaxy.

e Merging rate $\left(\mathrm{Gpc}^{-3} \mathrm{yr}^{-1}\right)$ given by Equation 28, assuming a constant number fraction of BBHs, i.e., $f_{n b}=\min \left(10^{-3}, 10^{-2} m_{\star} /\left\langle m_{\mathrm{BBH}}\right\rangle\right)$.

${ }^{f}$ Merging rate $\left(\mathrm{Gpc}^{-3} \mathrm{yr}^{-1}\right)$ given by Equation 28, assuming a constant mass fraction of BBHs, i.e., $f_{m b}=10^{-3}$.

${ }^{g}$ Percentage of BBHs with $e_{10 \mathrm{~Hz}}>0.01$ or $e_{10 \mathrm{~Hz}}>0.05$ (in the brackets) after all galaxies are summed.

In previous sections we have assumed that the BBHs are migrated into the nucleus cluster from the star forming regions outside the cluster. However, it is also possible that the BBHs are originated from the star formation processes within the cluster. For example, our MilkyWay center has an intense star formation process within the inner parsec (e.g., Figer et al. 2004). If so, the KL oscillations may be efficient in merging these BBHs. To cover these complexities, we explore cases where initially the BBHs are located at $r_{i}=r_{h}$ or $r_{i}=0.1 r_{h}$.

We assume that the initial inner orbital period distribution of the $\mathrm{BBHs}$ is given by Figure 2 of Belczynski et al. (2004). The period ranges from 1 to $10^{6}$ days. To reduce the signals of GW merging rates of the BBHs that have evolved in isolation, without any impacts from dynamical effects in the galactic nucleus, we require that initially the GW orbital decay timescale of each $\mathrm{BBH}$ is larger than $1000 \mathrm{Myr}$, i.e., $T_{\mathrm{GW}}>1000 \mathrm{Myr}$. Thus, our estimation of the event rates in this section is likely a conservative one.

The explored four different models are shown in Table 5 . For simplicity, we assume $\alpha_{\star}=7 / 4$ for these models. Using the method discribed in Section 2, we perform numerical simulations and obtain the merge event rates for each model for $\mathrm{MBH}$ with mass ranging from $10^{5} \mathrm{M}_{\odot}$ to $10^{9} M_{\odot}$.

\subsection{The merging event rates of BBHs}

The results of normalized merging rates for single galaxies are shown in Table 5 . We can see that $\mathscr{R}$ is a decreasing function of $M_{\bullet}$, and $m_{\star}$ (see also the top panel of Figure 11). This is mainly because the larger the mass of the central MBH, and the mass of the field stars, the softer the BBHs becomes, and thus the easier for them to be disrupted due to binary-single encounters. On the other hand, the tidal radius increases with the MBH mass, thus, the BBHs will be easier to be disrupted around massive MBHs. Figure 12 shows the 

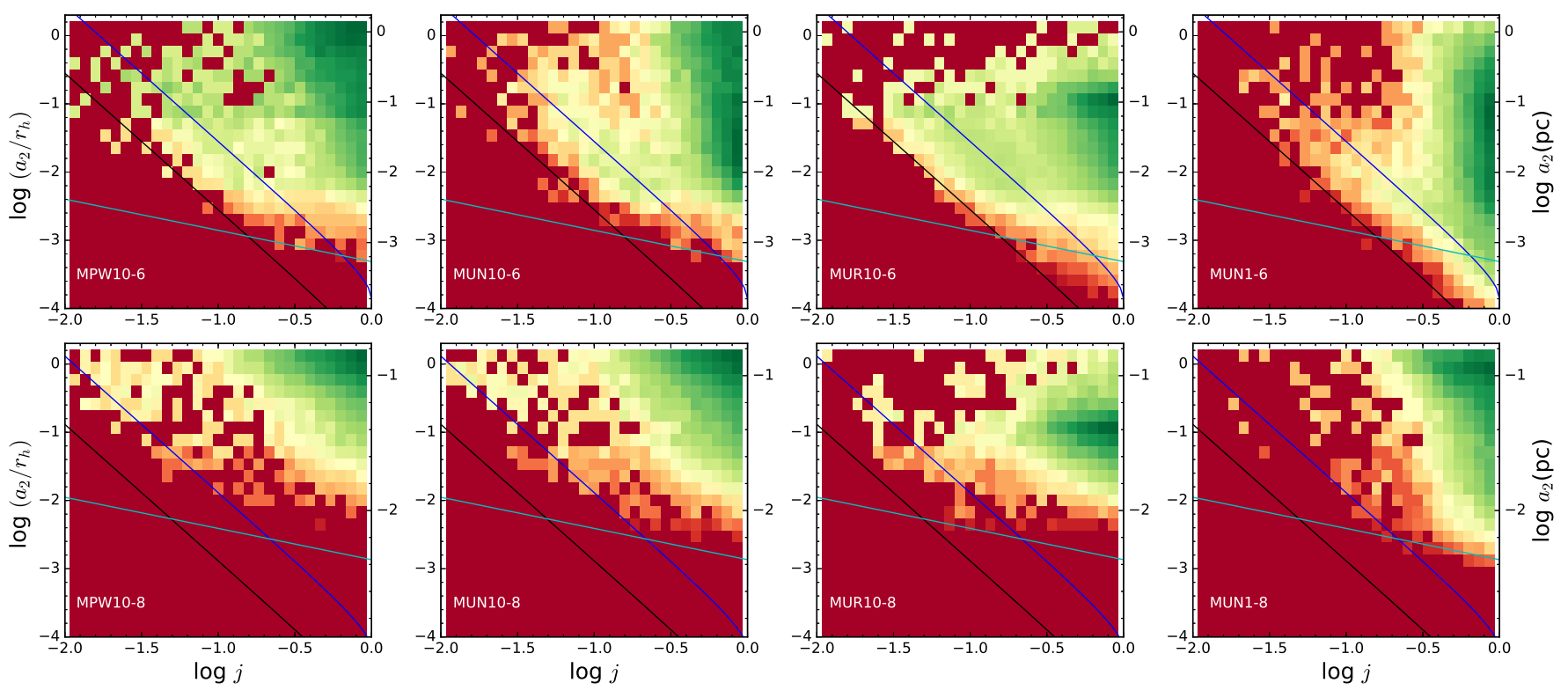

Figure 12. The density distribution of $a_{2}-j$ where the BBHs merged in different models. Here $j$ is the dimensionless angular momentum $j=\sqrt{1-e_{2}^{2}}$. The blue (black) lines in each panel show the tidal radius for a BBH with $a_{2}=0.5 \mathrm{AU}\left(a_{2}=0.05 \mathrm{AU}\right)$ and $m_{A}=m_{B}=5 M_{\odot}$. The green (red) color mesh means high (low) number density regions. The cyan solid lines in all panels show the locus of SB.

distribution of the position where the BBHs merged. We can see that for massive $\mathrm{MBH}$, the BBH only merges at the outskirt of the nuclei, but for a MBH with a smaller mass, the BBH can merge in the inner parts of the cluster. Given the same MBH, if the mass of field stars is smaller, the BBHs can reach to deeper regions of the cluster.

To estimate the total event rate in nearby universe, we need to integrate the merge events over all galaxies. The number density of MBHs in the universe is given by (Aller \& Richstone 2002)

$$
\frac{d n}{d M_{\bullet}}=c_{0}\left(\frac{M_{\bullet}}{m_{0}}\right)^{-1.25} \exp \left(-\frac{M_{\bullet}}{m_{0}}\right)
$$

where $c_{0}=3.2 \times 10^{-11} M_{\odot}^{-1} \mathrm{Mpc}^{-3}$ and $m_{0}=1.3 \times$ $10^{8} M_{\odot}$. The cosmological event rate is then

$$
R_{\mathrm{tot}}=\int_{10^{5} M_{\odot}}^{10^{9} M_{\odot}} R\left(M_{\bullet}\right) \frac{d n}{d M_{\bullet}} d M_{\bullet}
$$

Here $R$ is given by Equation 25 or 26, depending on the model assumption, and we have assumed negligible merging events from a nucleus cluster with $M_{\bullet}>10^{9} M_{\odot}$ (see Figure 11).

To obtain the total event rates by Equation 28, we need to expand the results of $\mathscr{R}$ shown in Table 5 to arbitrary MBH masses from $10^{5}$ to $10^{9} M_{\odot}$. For MPW10 and MUN10 model series, we have

$$
\log \left(\mathscr{R} / \mathrm{Gyr}^{-1}\right) \simeq-1.14-0.51 \log M_{7}+0.04\left(\log M_{7}\right)^{2},
$$

and

$$
\log \left(\mathscr{R} / \mathrm{Gyr}^{-1}\right) \simeq-0.83-0.61 \log M_{7}+0.038\left(\log M_{7}\right)^{2},
$$

respectively, where $M_{7}=M_{\bullet} / 10^{7} M_{\odot}$. For MUR10 and MUN1 model series, we have

$\log \left(\mathscr{R} / \mathrm{Gyr}^{-1}\right) \simeq-0.045-0.66 \log M_{7}+0.030\left(\log M_{7}\right)^{2}$,

and

$\log \left(\mathscr{R} / \mathrm{Gyr}^{-1}\right) \simeq-0.34-0.85 \log M_{7}+0.0024\left(\log M_{7}\right)^{2}$,

respectively.

The estimated total rates of $\mathrm{BBH}$ merging events are shown in the last two columns of Table 5. If assuming a constant number fraction of BBHs, given $r_{i}=r_{h}$ and $m_{\star}=10 M_{\odot}$, we find that the total merging rate is 1.6 $\mathrm{Gyr}^{-1}$ and $3.2 \mathrm{Gyr}^{-1}$ for models assuming PW and UN mass functions, respectively. However, the rate can be up to $\sim 31 \mathrm{Gyr}^{-1}$ for a UN model and given $m_{\star}=$ $1 M_{\odot}$. Assuming a small $m_{\star}$ can increase the merging rates as the BBHs become harder in the cluster and can survive in inner regions of the cluster after multiple binary-single encounters. The merging event rates can also be increased to $\sim 20 \mathrm{Gyr}^{-1}$ for a UN model but given $r_{i}=0.1 r_{h}$ and $m_{\star}=10 M_{\odot}$. As the BBHs are initially located at distance much closer to the $\mathrm{MBH}$, the KL effects become much more effective in merging these BBHs, resulting in signficant increase of the merging rates. In this models, we find that the contribution of merging rates from KL oscillations is much larger than 

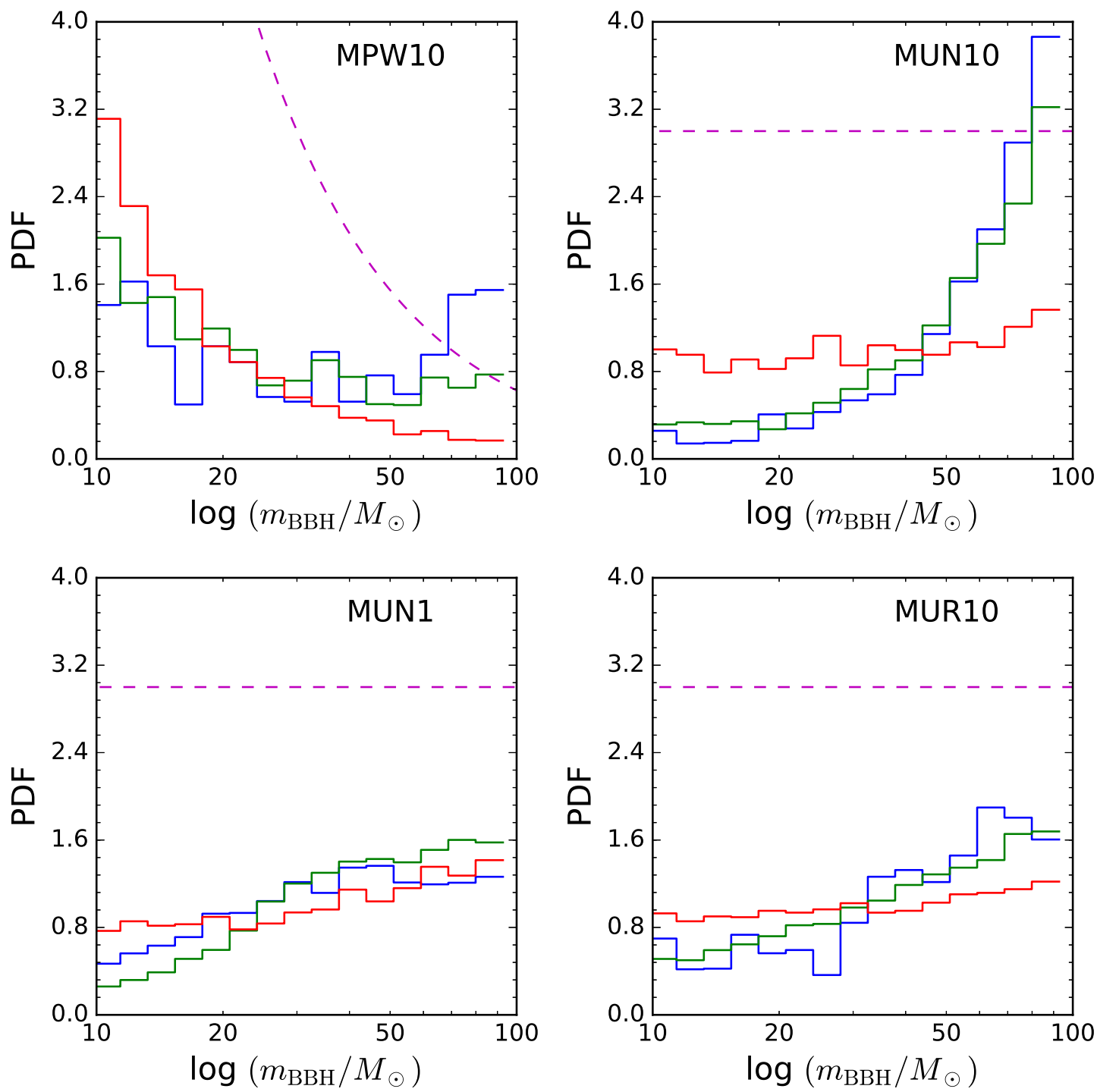

Figure 13. Distribution functions of the total mass of the merged BBHs in different models. The magenta dashed lines show the initial mass distribution of BBHs. Blue, green and red solid lines show the results for galaxies with $\mathrm{MBH}$ of mass $10^{5} M_{\odot}$, $10^{7} M_{\odot}$ and $10^{9} M_{\odot}$, respectively.

those from binary-single encounters.

If assuming a constant mass fraction of BBHs, the rates will be dramatically reduced. This is because $\left\langle m_{\mathrm{BBH}}\right\rangle$ is usually about $20 M_{\odot}-40 M_{\odot}$, depending on the assumed mass function (see in Table 5). Such a mass is usually larger (or much larger) than the mass of the field stars, and reduces significantly the number of BBHs in each galaxy. Nevertheless, the event rate is in orders of $1-10 \mathrm{Gpc}^{-3} \mathrm{yr}^{-1}$, which is still not negligible for LIGO detections. These results suggest that the $\mathrm{BBH}$ mergers in the center of galaxies can contribute partially to the LIGO observations.

We notice that for MBHs with $10^{8}-10^{9} M_{\odot}$, the two body relaxation timescale in the cluster is much longer than the Hubble timescale. Thus, in reality these cluster may never reach the equilibrium state. Our estimated merging rates for these clusters could be problematic. Nevertheless, the merging rates contributed from these galaxies are small (See Figure 11), and our estimations of the total merging rates should not be significantly affected.

\subsection{The mass and eccentricity distribution of the merging $\mathrm{BBHs}$}

Figure 13 shows the mass distributions of the merged $\mathrm{BBH}$ in different models. We can see that, compared with the initial mass distribution (the dashed magenta lines in each panel), the merged BBHs are likely more massive. For galaxies containing smaller MBHs with $M_{\bullet} \lesssim 10^{7} M_{\odot}$, the merged BBHs are also likely more 

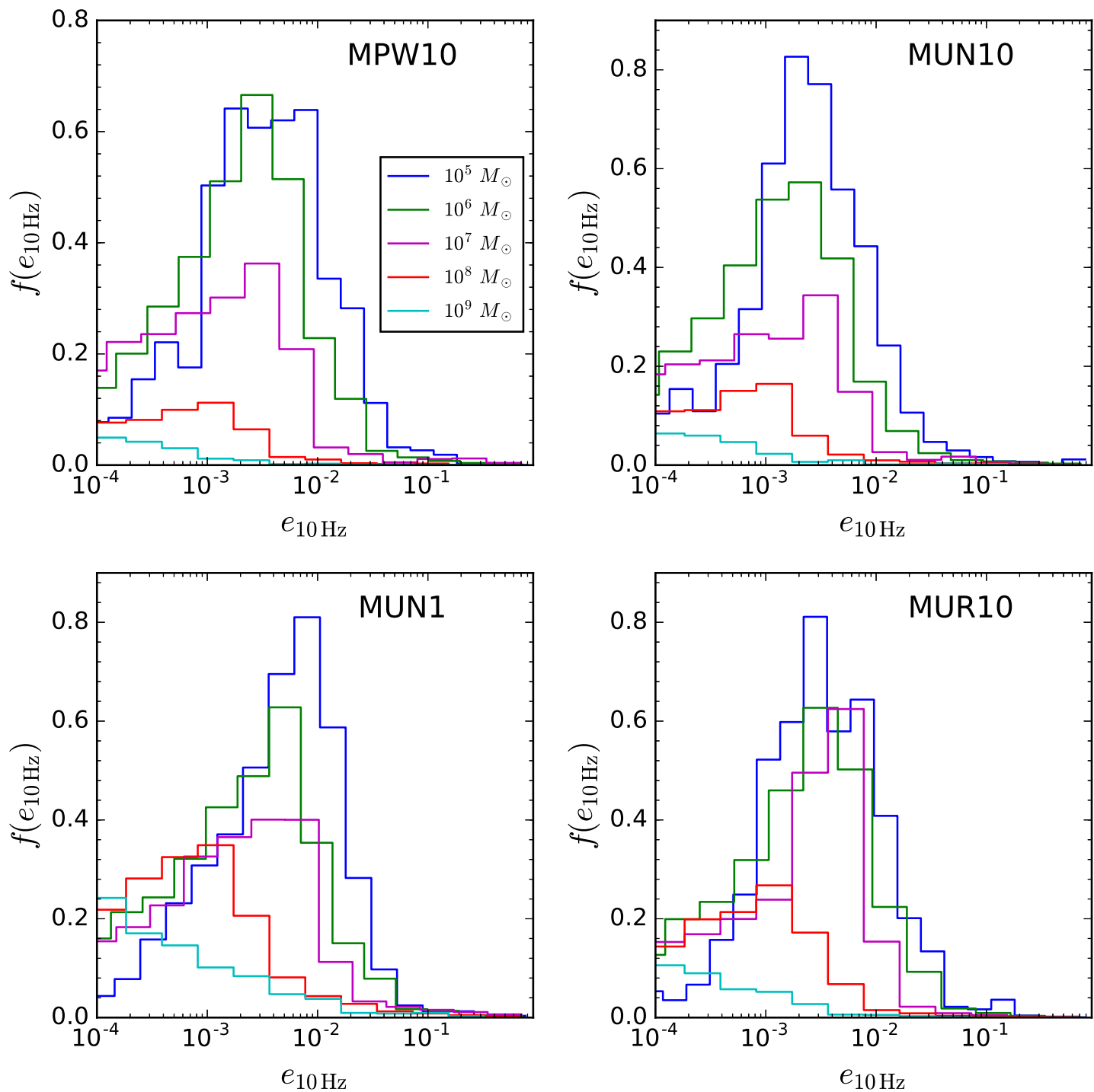

Figure 14. The distribution of eccenctricity of BBHs when its peak $\mathrm{GW}$ frequency enters the LIGO band, i.e., $f_{\mathrm{GW}}=10 \mathrm{~Hz}$. Different panels show results for different models in Table 5. The solid lines in different colors in each panel show the results when the mass of MBH in the cluster is different (see the legend in the left panel).

massive than those galaxies containing larger $\mathrm{MBHs}$ with $M_{\bullet} \gtrsim 10^{8} M_{\odot}$. More massive BBHs can survive longer in the galaxies with smaller MBHs, and thus they have higher event rates for LIGO. Such preference for massive BBHs is less significant if the BBHs can penetrate into the inner regions of the cluster, as both the massive and less massive BBHs can be quickly merged in the inner regions, as shown in the bottom panels of Figure 13 (for models MUN1 and MUR10).

Figure 14 shows the distribution of the eccentricity of the BBHs when the GW frequency approaches $f_{\mathrm{GW}}=10 \mathrm{~Hz}$. We can see that the eccentricity for low mass MBHs are relatively higher than those around massive MBHs. For MBH with mass $10^{5} M_{\odot}$, we find that $\sim 10-20 \%$ of the merged BBHs have $e_{10 \mathrm{~Hz}}>0.01$.
Many BBHs are hard in these cluster and they are likely more compact after each binary-single encounter. Thus, the eccentricity of them in the merging phase can be high. On the contrary, around massive MBHs the merged BBHs are commonly less eccentric as many of them are soft binaries, and that a significant number of them are merging by evolving in isolation. If the $\mathrm{MBH}$ is more massive, i.e., $10^{9} M_{\odot}$, the fraction will be about $\lesssim 1 \%$. We also find that if the BBHs are initially located at inner regions, e.g., $r_{i}=0.1 r_{h}$ in model MUR10 series, the merging eccentricities can be significantly higher, as the KL oscillations can be much more effective.

The percentage of $\mathrm{BBH}$ mergers with $e_{10 \mathrm{~Hz}}>0.01$ will be around $3-10 \%$ for all galaxies in the local universe (see the last column of Table 4). As comparison, 
the expected percentage of $e_{10 \mathrm{~Hz}}>10^{-3}$ in globular clusters is $\sim 1 \%$ (Rodriguez et al. 2016b). For BBH mergers from galactic fields, the final merging eccentricity is practically zero (Peters 1964; Belczynski et al. 2016). Thus in principle they can be distinguished from $\mathrm{BBH}$ mergers from the galactic fields and globular clusters using eccentric waveforms when enough events are accumulated (Hinderer \& Babak 2017).

\section{DISCUSSION}

Galactic nucleus cluster is a very complex system that multiple components of objects, including the stars, white dwarfs, neutron stars, black holes, and the binaries combined by any of these objects, are interacting with the central $\mathrm{MBH}$ and each other across the evolution history of galaxies. Although we have included many dominating dynamical effects, we warn the readers that there are still a number of complexities that we have not included in our simulation, which may affect further the merging rate and the dynamical evolutions of the BBHs.

- We have assumed the field stars with a single mass, while they should follow a spectrum of mass distributions in reality. Due to the mass segregation effect, massive stars/objects are concentrated in inner regions while those less massive ones are more likely at the outer parts. Thus, the dynamics of BBHs in the outer parts of the cluster may be different from the inner parts. Additionally, we do not trace and discuss those BBHs if one of its components is exchanged by the field stars, and we do not consider the case that the isolated black holes in the cluster can be captured into binary systems. These complexities can be considered in the future, if we can simulate the relaxation process by methods similar to those in Hénon (1971) and Joshi et al. (2000).

- In estimating the merging event rates, we have assumed a constant mass or number ratio of stellarmass BBHs with respect to the background field stars. In reality, neither of these two cases may be true, as the number of BBHs may vary from galaxies to galaxies, and may depend on various properties of the nucleus cluster and the black holes.

- We have assumed that the BBHs formed continuously inside or outside of the cluster, as suggested by the continuous star formation history observed in the nuclear star cluster in many galaxies (e.g., Walcher et al. 2006). This assumption may have over simplified the complex star formation history of nuclear star clusters under the cosmological context, where the infalling gas due to the merging of galaxies, migration of stellar clusters and the existence of binary MBHs may affect the supply of BBHs (e.g., Antonini et al. 2015; Arca-Sedda \& Gualandris 2018; Arca-Sedda \& Capuzzo-Dolcetta 2019).

Our treatment of the vector RR process may be oversimplified. However, even with a more sophisticated treatment, e.g., given by Hamers et al. (2018), they find that the vector $R R$ enhances the merger rate only with MBHs of small masses, and drops sharply with increasing MBH masses. Thus, we consider that the details have no significant effects on our results.

We have not included the precession of the outer orbit due to the distributed mass when calculating the KozaiLidov oscillations in the current simulation. To explore the impact on our results due to such simplification, we perform additional simulations that include such precession of the outer orbit. By comparing the simulation results, we find only a slight reduce on the merging rates from Kozai-Lidov mechanism, and our main results on the merging rates will not be significantly affected. This is consistent with those in Hoang et al. (2018).

Our work can be expanded to consider the evolution and the merging of neutron star binaries, or the neutron star-black hole binaries. Currently it is still difficult to distinguish the merging channels of BBHs as their localizations are challenging, as there are no definite electromagnetic counterparts observed for these events. However, the merging of neutron star binaries or neutron star-black hole binaries in galactic nucleus are supposed to have electromagnetic counterparts (Abbott et al. 2017d) and their localizations can be quite accurate. If these events can be detected by LIGO and that its origin can be confirmed by their electromagnetic counterparts or the measurement of the eccentricity, their properties can be used further to study the dynamics around the MBHs. We will defer such studies to future.

There are a number of important difference between the evolutions of BBHs that are located in the globular clusters and the galactic nuclei. For example, most of the $\mathrm{BBHs}$ residing in globular clusters are hard binaries, and every binary-single encounters are likely to harden them. The hardening of BBHs will usually eject them out of the globular cluster, and thus their mergers are likely taking place outside of the globular cluster. However, in a galactic nucleus, the BBHs are most likely soft binaries, and they are likely be softened and ionized by the binary-single encounters. On the other hand, if they merge, they merge within the galactic nucleus. In a galactic nucleus, there are unique dynamical processes, for example, the resonant relaxations, the KL effects and 
tidal disruptions of BBHs by the central MBHs. Thus, the event rate and statistical properties of their GW signals will likely be different from those of the globular clusters. By comparing their difference, we may likely distinguish the GW events in these two scenarios with future observations.

Although we find that our Monte Carlo scheme can reproduce the theoretical expectations of individual dynamical effects, we still can not guarantee that the combinations of these effects have well captured the detailed evolutions of the BBHs around MBHs. Thus, our numerical method still needs verifications (or calibrations) by $N$-body simulations that can combine well of the dynamical effects that we have considered. Though rewarding, these $N$-body simulations are very challenging and expensive, which are out of the scope of this study. We defer it to future investigations.

\section{CONCLUSIONS}

One of the possible channels to merge the stellar mass binary black holes (BBHs) is in a galactic nucleus that contains a $\mathrm{MBH}$ in the center. In this work, we study the dynamical evolution of the $\mathrm{BBHs}$ in a galactic nucleus that contains a $\mathrm{MBH}$. Along the pathway of their final merging, we consider simultaneously the non-resonant and resonant relaxations of the $\mathrm{BBHs}$, the binary-single encounters of the BBHs with the field stars, the KozaiLidov (KL) oscillation and the close encounters between the BBHs and the central MBH, which usually lead to BBHs' tidal disruptions. These effects have been individually studied and discussed in lots of previous work, however, not yet well combined. Here we consider all of them in a Monte-Carlo scheme to study the merging of $\mathrm{BBHs}$ for a comprehensive study.

We find that the tidal disruption of BBHs by MBHs can effectively reduce the number of the BBHs in the inner regions of a nucleus cluster, especially if resonant relaxation (RR) are being considered. The binary-single encounters can further reduce the number of $\mathrm{BBHs}$ within the cluster, as most of the BBHs become soft binaries. Each encounter can possibly increase the separations of the BBHs and cumulatively lead to the ionization of the BBHs. In the meanwhile, the binary-single encounters can also make the binary hardening, and in- crease the eccentricity of the BBHs. These effects can increase the merging rates significantly.

If the total mass of the $\mathrm{BBH}$ is heavier than the mass of the field stars, they can sink to the central regions by mass segregation effects, making the KL oscillation an important factor to trigger their mergers. For other dynamical effects, for example, the vector RR process, we do not see significant difference of the merging rates with or without it.

We find that in our simulations the mergers of BBHs are mainly contributed by galaxies containing MBHs that are less massive than $10^{8} M_{\odot}$, and the total event rates are likely in orders of $1-10 \mathrm{Gpc}^{3} \mathrm{yr}^{-1}$, depending also on the detailed assumptions of the nucleus clusters. The eccentricity of the BBHs $\left(e_{10 \mathrm{~Hz}}\right)$ when its peak $\mathrm{GW}$ frequency enters into the observational bands of the Advanced LIGO/Virgo detectors (e.g., $10 \mathrm{~Hz}$ ) depends on the initial conditions of the cluster. It is likely that there are $3-10 \%$ of BBHs with $e_{10 \mathrm{~Hz}}>0.01$, and the probability is higher for clusters harboring $\mathrm{MBHs}$ with a smaller mass. This will be a smoking-gun signal when solid statistical analysis becomes available with accumulating events. Our results show that the mergers of BBHs within galactic nuclei can be one of the important sources of the merging events detected or to be detected by ground-based GW detectors.

We thank the anonymous referee for the helpful comments that have improved this paper. This work was supported in part by the National Natural Science Foundation of China under grant No. 11603083, 11673077. This work was also supported in part by Guangzhou university Startup funds grand No. 69-18ZX10362, "the Fundamental Research Funds for the Central Universities" grant No. 161GPY51, the Key Project of the National Natural Science Foundation of China under grant No. 11733010. LS was supported by the Young Elite Scientists Sponsorship Program by the China Association for Science and Technology (2018QNRC001), and partially supported by the National Natural Science Foundation of China (11721303), XDB23010200. The simulations in this work are performed partly in the TianHe II National Supercomputer Center in Guangzhou, and partially on the computing cluster in School of Physics and Astronomy, Sun Yat-Sen University.

\section{REFERENCES}

Abbott, B. P., Abbott, R., Abbott, T. D., et al. 2016a, Physical Review Letters, 116, 061102

Abbott, B. P., Abbott, R., Abbott, T. D., et al. 2016b, ApJL, 833, L1

Abbott, B. P., Abbott, R., Abbott, T. D., et al. 2016c, Physical Review Letters, 116, 241103
Abbott, B. P., Abbott, R., Abbott, T. D., et al. 2016, Physical Review X, 6, 041015

Abbott, B. P., Abbott, R., Abbott, T. D., et al. 2017a, Physical Review Letters, 118, 221101

Abbott, B. P., Abbott, R., Abbott, T. D., et al. 2017b, ApJL, 851, L35 
Abbott, B. P., Abbott, R., Abbott, T. D., et al. 2017c, Physical Review Letters, 119, 141101

Abbott, B. P., Abbott, R., Abbott, T. D., et al. 2017d, ApJL, 848, L12

The LIGO Scientific Collaboration, the Virgo Collaboration, Abbott, B. P., et al. 2018, arXiv:1811.12907

Antonini, F., Faber, J., Gualandris, A., \& Merritt, D. 2010, ApJ, 713, 90

Antonini, F., \& Merritt, D. 2013, ApJL, 763, L10

Antonini, F., Barausse, E., \& Silk, J. 2015, ApJ, 812, 72

Antonini, F., \& Perets, H. B. 2012, ApJ, 757, 27

Antonini, F., \& Rasio, F. A. 2016, ApJ, 831, 187

Alexander, T. 2005, PhR, 419, 65

Alexander, T., \& Hopman, C. 2009, ApJ, 697, 1861

Aller, M. C., \& Richstone, D. 2002, AJ, 124, 3035

Arca-Sedda, M., \& Gualandris, A. 2018, MNRAS, 477, 4423

Arca-Sedda, M., \& Capuzzo-Dolcetta, R. 2019, MNRAS, 483, 152

Bahcall, J. N., \& Wolf, R. A. 1976, ApJ, 209, 214

Bahcall, J. N., \& Wolf, R. A. 1977, ApJ, 216, 883

Bar-Or, B., \& Alexander, T. 2016, ApJ, 820, 129

Belczynski, K., Sadowski, A., \& Rasio, F. A. 2004, ApJ, 611, 1068

Belczynski, K., Sadowski, A., Rasio, F. A., \& Bulik, T. 2006, ApJ, 650, 303

Belczynski, K., Holz, D. E., Bulik, T., \& O'Shaughnessy, R. 2016, Nature, 534, 512

Binney, J., \& Tremaine, S. 1987, Princeton, NJ, Princeton University Press, 1987, 747 p.,

Blaes, O., Lee, M. H., \& Socrates, A. 2002, ApJ, 578, 775

Cohn, H., \& Kulsrud, R. M. 1978, ApJ, 226, 1087

Downing, J. M. B., Benacquista, M. J., Giersz, M., \& Spurzem, R. 2010, MNRAS, 407, 1946

Dominik, M., Berti, E., O'Shaughnessy, R., et al. 2015, ApJ, 806, 263

Dormand, J. R., \& Prince, P. J. 1980, J. Comp. Appl. Math., Vol.6, p.19

Hairer, E., Norsett, S. P., \& Wanner, G. 1987, Solving Ordinary Differential Equations I. Nonstiff Problems, Springer Series in Comput. Mathematics, Vol. 8 (Springer-Verlag)

Figer, D. F., Rich, R. M., Kim, S. S., Morris, M., \& Serabyn, E. 2004, ApJ, 601, 319

Ford, E. B., Kozinsky, B., \& Rasio, F. A. 2000, ApJ, 535, 385

Fragione, G., Grishin, E., Leigh, N. W. C., Perets, H. B., \& Perna, R. 2018, arXiv:1811.10627

Fragione, G., \& Kocsis, B. 2018, Physical Review Letters, 121, 161103

Grishin, E., Perets, H. B., \& Fragione, G. 2018, MNRAS, 481, 4907

Hamers, A. S., Bar-Or, B., Petrovich, C., \& Antonini, F. 2018, arXiv:1805.10313
Heggie, D. C., \& Hut, P. 1993, ApJS, 85, 347

Hénon, M. H. 1971, Ap\&SS, 14, 151

Hinderer, T., \& Babak, S. 2017, Physical Review D, 96, 104048

Hopman, C., \& Alexander, T. 2006, ApJ, 645, 1152

Hopman, C. 2009, ApJ, 700, 1933

Hoang, B.-M., Naoz, S., Kocsis, B., Rasio, F. A., \& Dosopoulou, F. 2018, ApJ, 856, 140

Hut, P., \& Bahcall, J. N. 1983, ApJ, 268, 319

Kiseleva, L. G., Eggleton, P. P., \& Mikkola, S. 1998, MNRAS, 300, 292

Kormendy, J., \& Ho, L. C. 2013, ARA\&A, 51, 511

Kozai, Y. 1962, AJ, 67, 591

Joshi, K. J., Rasio, F. A., \& Portegies Zwart, S. 2000, ApJ, 540, 969

Lightman, A. P., \& Shapiro, S. L. 1977, ApJ, 211, 244

Magorrian, J., \& Tremaine, S. 1999, MNRAS, 309, 447

Mandel, I., Sesana, A., \& Vecchio, A. 2018, Class. Quantum Grav., 35, 054004

Merritt, D., Alexander, T., Mikkola, S., \& Will, C. M. 2011, PhRvD, 84, 044024

McMillan, S. L. W., \& Hut, P. 1996, ApJ, 467, 348

Miller, M. C., \& Lauburg, V. M. 2009, ApJ, 692, 917

Morscher, M., Pattabiraman, B., Rodriguez, C., Rasio, F. A., \& Umbreit, S. 2015, ApJ, 800, 9

Naoz, S., Farr, W. M., Lithwick, Y., Rasio, F. A., \& Teyssandier, J. 2013, MNRAS, 431, 2155

Naoz, S. 2016, ARA\&A, 54, 441

O'Leary, R. M., Kocsis, B., \& Loeb, A. 2009, MNRAS, 395, 2127

Peters, P. C. 1964, Phy. Rev., 136, 1224

Petrovich, C., \& Antonini, F. 2017, ApJ, 846, 146

Rauch, K. P., \& Tremaine, S. 1996, NewA, 1, 149

Rodriguez, C. L., Haster, C.-J., Chatterjee, S., Kalogera, V., \& Rasio, F. A. 2016, ApJL, 824, L8

Rodriguez, C. L., Chatterjee, S., \& Rasio, F. A. 2016, PhRvD, 93, 084029

Rodriguez, C. L., Morscher, M., Pattabiraman, B., et al. 2015, Physical Review Letters, 115, 051101

Shapiro, S. L., \& Marchant, A. B. 1978, ApJ, 225, 603

Samsing, J., MacLeod, M., \& Ramirez-Ruiz, E. 2014, ApJ, 784, 71

Sigurdsson, S., \& Phinney, E. S. 1993, ApJ, 415, 631

VanLandingham, J. H., Miller, M. C., Hamilton, D. P., \& Richardson, D. C. 2016, ApJ, 828, 77

Walcher, C. J., Böker, T., Charlot, S., et al. 2006, ApJ, 649, 692

Wen, L. 2003, ApJ, 598, 419

Zhang, F., Lu, Y., \& Yu, Q. 2010, ApJ, 722, 1744

\section{APPENDIX}

\section{A. THE DYNAMICS OF THE OUTER ORBITS}

\section{A.1. The diffusion coefficients in two-body relaxations}

The diffusion coefficients $D_{E E}$ and $D_{J J}$ describe the orbit-averaged scatterings of the energy and angular momentum of the orbit for point-like particles around the MBH. Similarly, $D_{E}$ and $D_{J}$ describe the orbit-averaged drifts of the energy and angular momentum, respectively, and $D_{E J}$ describes the correlations between the two. The calculations of the diffusion coefficients have been done and discussed in many studies (e.g., Shapiro \& Marchant 1978; Cohn \& Kulsrud 1978; Bar-Or \& Alexander 2016). Here we use the formalism in Bar-Or \& Alexander (2016) where the mass of the binary can be different from the field stars. Denote the SMA and the eccentricity of the particle cycling around the MBH as $a_{2}$ and $e_{2}$, and suppose that the dimensionless distribution function of the field stars is given by $f(E)$. Denote $m_{\mathrm{BBH}}$ and $m_{\star}$ as the masses of the binary and the field star respectively. Then firstly the function $\Gamma_{i j k}$ 
and $\Gamma_{0}$ are calculated,

$$
\begin{aligned}
\Gamma_{i j k} & =2^{1+k-i} \frac{\kappa}{\pi} \int_{-1}^{1} d y \int_{1}^{2 /(1+y)} d s f_{a}(s E) \\
& \times \frac{1}{\sqrt{1-y^{2}}} \frac{\left(1+y e_{2}\right)^{i}}{\left(v^{2} / E\right)^{k}}\left(v_{a} / v\right)^{j} \\
\Gamma_{0} & =\kappa \int_{-\infty}^{1} d s f_{a}(s E) .
\end{aligned}
$$

Here $\kappa=\left(4 \pi G m_{\star}\right)^{2} \ln \Lambda, y=\left(r / a_{2}-1\right) / e, v_{a}^{2}=2 E\left(2 a_{2} / r-s\right), v^{2}=2 E\left(2 a_{2} / r-1\right), \Lambda=M_{\bullet} / m_{\star}$. Then the diffusion coefficients are given by

$$
\begin{aligned}
D_{E} / E & =\frac{m_{\mathrm{BBH}}}{m_{\star}} \Gamma_{110}-\Gamma_{0}, \\
D_{E E} / E^{2} & =\frac{4}{3} \Gamma_{13-1}+\Gamma_{0}, \\
D_{J} /\left(j J_{c}\right) & =\frac{5-3 j^{2}}{12} \Gamma_{0}-j^{2} \frac{m_{\mathrm{BBH}}+m_{\star}}{2 m_{\star}} \Gamma_{111}+\Gamma_{310}-\frac{1}{3} \Gamma_{330}, \\
D_{J J} / J_{c}^{2} & =\frac{5-3 j^{2}}{6} \Gamma_{0}+\frac{j^{2}}{2} \Gamma_{131}-\frac{j^{2}}{2} \Gamma_{111}+2 \Gamma_{310}-\frac{2}{3} \Gamma_{330}, \\
D_{E J} /\left(E J_{c}\right) & =-\frac{2}{3} j\left(\Gamma_{0}+\Gamma_{130}\right) .
\end{aligned}
$$

Here $j=J / J_{c}$ is the dimensionless angular momentum, and $J_{c}=\sqrt{G M_{\bullet} a_{2}}$ is the maximum angular momentum. Note that $j$ could not be either zero nor one, otherwise the integration will be divergent.

\section{A.2. The scalar and the vector resonant relaxations}

The scalar and vector resonant relaxations change only the angular momentum of the outer orbit of the binary. For the scalar resonant relaxation, we take formalisms similar to Bar-Or \& Alexander (2016). Define $Q=M_{\bullet} / m_{\star}$ as the number of field stars and $\nu_{r}=2 \pi / P\left(a_{2}\right)=a_{2}^{-3 / 2} M_{\bullet}^{1 / 2} G^{1 / 2}$ as the Keplerian orbital frequency, the RR torque $\tau_{N}$ is given by

$$
\tau_{N}=0.28 \sqrt{1-j} \sqrt{N\left(2 a_{2}\right)} G m_{\star} / a_{2} .
$$

Here $N\left(a_{2}\right)=M_{\bullet} / m_{\star}\left(a_{2} / r_{h}\right)^{3-\alpha}$. Denote $\nu_{J}=\tau_{N} / J_{c}$, the diffusion coefficients are given by

$$
D_{J J}^{\mathrm{RR}}=\frac{2 J_{c}^{2} \nu_{J}^{2}(j) T_{c}\left(a_{2}\right)}{1+\left[T_{c}\left(a_{2}\right) \nu_{p}\left(a_{2}, j\right)\right]^{2}}
$$

Here

$$
\nu_{p}=\left|\nu_{M}+\nu_{\mathrm{GR}}\right|,
$$

is the precession frequency, where

$$
\nu_{\mathrm{GR}}=\frac{3 r_{g}}{a_{2} j^{2}} \nu_{r}\left(a_{2}\right)
$$

is the frequency of the GR-induced precession. $\nu_{M}$ has an exact form when $\alpha=2$,

$$
\nu_{\mathrm{M}}(\alpha=2, j)=-\frac{N\left(a_{2}\right)}{Q} \frac{j}{j+1} \nu_{r} .
$$

For other values, it can be obtained numerically. We find that

$$
\nu_{\mathrm{M}}(\alpha, j)=-\frac{N\left(a_{2}\right)}{Q} \frac{j^{2-\gamma}\left(1-j^{\gamma}\right)}{1-j^{2}} \nu_{r}
$$

When $\alpha=7 / 4, \gamma=1.48$; when $\alpha=1 / 2$, we find that $\gamma=4.0$; when $\alpha=2, \gamma=1$, which reduces to Equation A7; when $\alpha=1$, we find that $\gamma=3.37$.

$T_{c}\left(a_{2}\right)$ is given by (note that the coherence time $T_{c}$ here has not included the GR precession (Bar-Or \& Alexander 2016))

$$
T_{c}\left(a_{2}\right)=\sqrt{\pi / 2} \nu_{M}^{-1}\left(2 a_{2}, \sqrt{1 / 2}\right) .
$$


The drift term is given by

$$
D_{J}^{\mathrm{RR}}=\frac{1}{2 J} \frac{\partial\left(J D_{J J}^{\mathrm{RR}}\right)}{\partial J} .
$$

For the vector RR relaxtion, we take a very simple model, where the direction of the angular momentum is randomly walking on the sufrace of the sphere, with the unity change happens in a timescale given by $T_{\mathrm{RR}}^{v}$ in Equation 4 . We generate a random vector $\hat{d} j$ with magnitude $l=\left(d t / T_{\mathrm{RR}}^{v}\right)^{1 / 2}$, where $l$ is the standard deviation of a log-normal distribution. The unit angular momentum of the CM is changed to $\hat{j}^{\prime}=\hat{j}+\hat{d j}$; the direction of $\hat{d j}$ is set such that $\hat{j}^{\prime}$ is always a unit vector. Here $\hat{j}$ is the unit angular momentum. First we solve a reference vector $\hat{d j}_{0}=(0, l \cos s, l \sin s)$ where $\left|\hat{j}+\hat{d} j_{0}\right|=1$ (If $j_{y}^{2}+j_{z}^{2}<l^{2} / 4$, we can set $\hat{d} j_{0}=(l \cos s, 0, l \sin s)$ ). Then the random vector $\hat{j}^{\prime}$ is generated after rotating $\hat{j}+\hat{d j}_{0}$ around the vector $\hat{j}$ by a random angle $\phi \in(0,2 \pi)$. If $l>1$, we simply set the angular momentum randomly distributed.

\section{B. THE GW FREQUENCY OF THE MERGING BBHS}

The power of GWs emitted from an eccentric in-spiraling BBH covers a broad range of frequency and the maximum occurs at the peak frequency $f_{\mathrm{GW}}$, which is given by (Wen 2003)

$$
f_{\mathrm{GW}}=\frac{\sqrt{\left(m_{A}+m_{B}\right) G}}{\pi} \frac{\left(1+e_{1}\right)^{1.1954}}{a_{1}^{3 / 2}\left(1-e_{1}^{2}\right)^{3 / 2}} .
$$

If the BBHs are not affected by the KL effects, during their merging process, the evolution of $a_{1}$ and $e_{1}$ are given by (Peters 1964)

$$
a_{1}\left(e_{1}\right)=\frac{c_{0} e_{1}^{12 / 19}}{1-e_{1}^{2}}\left(1+\frac{121}{304} e_{1}^{2}\right)^{870 / 2299} .
$$

Here $c_{0}$ is a constant determined by the initial parameters.

In our simulation, we mainly focus on the eccentricity of the BBHs when $f_{\mathrm{GW}}=10 \mathrm{~Hz}$, i.e., $e_{10 \mathrm{~Hz}}$. When a $\mathrm{BBH}$ can be considered as a merger in the simulation (see Section 2.5), $e_{10 \mathrm{~Hz}}$ is calculated according to the details of the simulation: (1) During the merge, if the BBHs are not affected by the KL effects, we obtain the value of $e_{10 \mathrm{~Hz}}$ by setting $f_{\mathrm{GW}}=10 \mathrm{~Hz}$ in Equation B11 and combining with Equation B12. The constant $c_{0}$ is determined according to the last value of $e_{1}$ and $a_{1}$ of the BBHs. (2) If the BBHs are affected currently by KL effects and that KL term dominates over the $\mathrm{GW}$ term, i.e., $\left|\dot{e}_{1}^{\mathrm{KL}}\right|>\left|\dot{e}_{1}^{\mathrm{GW}}\right|$, we then record the value of $e_{1}$ as $e_{10 \mathrm{~Hz}}$ when $f_{\mathrm{GW}}=10 \mathrm{~Hz}$. (3) If the BBHs are affected currently by KL effects and that GW term dominates over the KL term, i.e., $\left|\dot{e}_{1}^{\mathrm{KL}}\right|<\left|\dot{e}_{1}^{\mathrm{GW}}\right|$, we calculate the value of $e_{10 \mathrm{~Hz}}$ similar to (1).

\section{THE BINARY-SINGLE ENCOUNTERS}

Here we first calculate the rates of binary-single encounters in a nucleus cluster. Suppose that the density profile of the field stars is stable, then the rate of binary-single encounters is given by (Sigurdsson \& Phinney 1993)

$$
R_{\mathrm{EC}}\left(a_{2}\right)=\left\langle n(r) \Sigma v_{\infty}\right\rangle=\frac{2}{P} \iint_{r_{p}}^{r_{a}} n\left(r, v^{\prime}\right) \Sigma v_{\infty}\left(a_{2}\right) \frac{d r}{v_{r}} d^{3} v^{\prime}
$$

where $\Sigma$ is the cross section of binary-single encounter. $v_{\infty}=\left|\vec{v}-\vec{v}^{\prime}\right|$ is the relative velocity between the binary and the star. $v=2\left(E+G M_{\bullet} / r\right), v_{r}, r$ and $a_{2}$ is the velocity, radial velocity, position and outer SMA of the binary respectively. $r_{a}=a_{2}\left(1+e_{2}\right)$ and $r_{p}=a_{2}\left(1-e_{2}\right)$ is apocenter and pericenter of the outer orbit, respectively.

The cross section for percienter passage less than $p$ is given by

$$
\Sigma=\pi p^{2}\left(1+\frac{2 m_{T} G}{p v_{\infty}^{2}}\right)
$$

Assuming that the velocity is isotropic, then we have $n\left(v^{\prime}, r\right)=n\left(v^{\prime}\right) n(r)$, where $n(r)=n_{0}\left(r / r_{h}\right)^{-\alpha_{\star}}$ and $n\left(v^{\prime}\right)$ satisfies a Maxiwell velocity distribution, with the velocity dispersion $\sigma_{a}$ satisfying (Alexander 2005)

$$
\sigma_{a}^{2}=\frac{1}{1+\alpha_{\star}} \frac{G M_{\bullet}}{r}
$$

The integration on $v^{\prime}$ can be preformed independently

$$
\left\langle v_{\infty}\right\rangle=\int n\left(v^{\prime}\right) v_{\infty} d^{3} v^{\prime}=\frac{2 \sigma_{a}}{(2 \pi)^{1 / 2}} \exp \left(-\frac{v^{2}}{2 \sigma_{a}^{2}}\right)+\left(v+\frac{\sigma_{a}^{2}}{v}\right) \operatorname{erf}\left(\frac{v}{\sqrt{2} \sigma_{a}}\right),
$$


and (Binney \& Tremaine 1987)

$$
\left\langle\frac{1}{v_{\infty}}\right\rangle=\int \frac{n\left(v^{\prime}\right)}{v_{\infty}} d^{3} v^{\prime}=\frac{1}{v} \operatorname{erf}\left(\frac{v}{\sqrt{2} \sigma_{a}}\right)
$$

We denote

$$
\Theta=\frac{2}{P} \int_{r_{p}}^{r_{a}}\left(\frac{r}{a_{2}}\right)^{-\alpha_{\star}} \frac{\left\langle v_{\infty}\right\rangle}{\sigma_{a}} \frac{d r}{v_{r}}
$$

and

$$
\Phi=\frac{2}{P} \int_{r_{p}}^{r_{a}}\left(\frac{r}{a_{2}}\right)^{-\alpha_{\star}} \sigma_{a}\left\langle\frac{1}{v_{\infty}}\right\rangle \frac{d r}{v_{r}} .
$$

Here $\Theta$ and $\Phi$ are functions of $e_{2} \alpha_{\star}$ only. By numerical simulations, when $\alpha_{\star}=7 / 4$, we find that $\Theta \simeq 1.36+0.49 e_{2}^{2}$ and $\Phi \simeq 0.86+2.5 e_{2}^{2}$; when $\alpha_{\star}=1$, we have $\Theta \simeq 1.47, \Phi \simeq 0.84+1.1 e_{2}^{2}$.

Then $R_{\mathrm{EC}}$ can be rewritten as

$$
R_{\mathrm{EC}}\left(a_{2}\right)=\pi p^{2} n_{0} \sigma_{a}\left(\frac{a_{2}}{r_{h}}\right)^{-\alpha_{\star}} \Theta+\frac{2 \pi p m_{T} G n_{0}}{\sigma_{a}}\left(\frac{a_{2}}{r_{h}}\right)^{-\alpha_{\star}} \Phi .
$$

Then the probability of taking $k$ times of binary-single encounters in time interval $\delta t$ is given by the Poisson distribution

$$
P_{\mathrm{EC}}(k)=e^{-\lambda} \frac{\lambda^{k}}{k !}
$$

where $\lambda=R_{\mathrm{EC}} \delta t$.

Occasionally, the binary will encounter with the single star with distance less than $0.01 \mathrm{AU}$. In these cases, the binary will experience an extremely strong encounter. However, if the incoming single object is a star, then the black hole touches, almost, to the surface of the star, leading to modifications of the trajectory due to tidal effects. If the incouming object is a black hole, then the gravitational wave can lead to the decay of the orbital energy, too. To avoid the complexities of these cases, we set a softening radius of $10^{-3} \mathrm{AU}$ between the incoming star and the black holes in the 3-body calculations.

Currently, we have neglected any relativistic effects on the binary-single encounters to reduce the computational costs. In principle, if the incoming object is a stellar mass black hole, we need to additionally consider the postNewtonian corrections of the orbit to include high order relativistic effects such as spin effects or the GW orbital decay. Such simplification may result in under estimation of the merging event rates. Considering that the fraction of stellar mass black holes in a nuclear cluster should be very small, e.g., $<10^{-2}$, the probability of such BBH-single black hole encounters should be small, and thus the impact on the merging event rates should not be significant. Nevertheless, we can include the post-Newtonian corrections into our 3-body simulations in future studies.

The initialization and termination of simulations of the binary-single encounters are considered as follows: (1) Initially the mass center of $\mathrm{BBH}$ and the single star are separated at a distance of $r_{b i}=50 a_{1}$ away from each other, where $a_{1}$ is the inner SMA of the BBH; (2) The mass center of BBH and the single star form a two body system. Denote $a_{2}^{\prime}=-m_{T} / v_{\infty}^{2}$ as the semimajor axis (SMA) of the orbit of this two body system and $\mathcal{M}$ as the mean anomaly of the $\mathrm{BBH}$ at distance $r_{b i}$, where $m_{T}$ is the total mass of $\mathrm{BBH}$-single star system and $v_{\infty}$ is the relative infinite velocity between the binary and the single star. Then we perform the 3-body simulation for a duration $d t=2|\mathcal{M}| /(2 \pi)\left(\left|a_{2}^{\prime 3}\right| / m_{T}\right)^{1 / 2}$. In a two-body problem, after such duration the mass center of BBHs will take one pericenter passage and return to the distance of $50 a_{1}$ again; (3) We determine the outcome of the BBH-single encounter. If we find that it is a fly-by, ionization or exchange event, the simulation stops; (4) However, if it is not any of the outcome in (3), usually the 3-body either form a triple system, or the 3-body are still in chaotic orbits, and we continue the simulations for additional time $d t \rightarrow 1.5 d t$ until it becomes any results in (3). We repeat (4) for at most 10 times such that the total time of simulations can be $\sim 170$ times of the original value of $d t$ in (2). In most cases, the binary will end up with one of the results listed in (3), but if not, we simply abandon the event and remove the BBH from the Monte-Carlo simulation.

When there are multiple encounters, i.e., $n_{\mathrm{EC}}>1$ in one time step $\delta t$ of our simulation (See Section 2.5 and 2.6), we assume that the first one of them occur within time of $\delta t / n_{\mathrm{EC}}$. After the first encounter, if it's a flyby event, the SMA and the eccentricity of the binary is changed. In the rest of the time step, i.e., $\delta t\left(n_{\mathrm{EC}}-1\right) / n_{\mathrm{EC}}$, we calculate the collision rate and the expected number of collisions, i.e., $n_{\mathrm{EC}}^{\prime}$, according to the updated orbits of the binary, and perform a successive encounter if $n_{\mathrm{EC}}^{\prime} \geq 1$. This repeats until there is no more successive encounters in the remain time of $\delta t$. 
Currently, we have ignored the correction of the outer orbit due to the binary-single encounter. The conversion between the energy of the inner and outer orbits during the binary-single encounter should be in orders of $\Delta E \sim \epsilon$, where $\epsilon$ is the energy of the inner orbit. Such simplification should not lead to significant differences as $\epsilon$ is usually much smaller than the energy of the outer orbit of the BBHs. Nevertheless, we will introduce corrections on both the energy and angular momentum of the outer orbits due to binary-single encounters in the future. 